\title{
Inclusive search for highly boosted Higgs bosons decaying to bottom quark-antiquark pairs in proton-proton collisions at $\sqrt{s}=13 \mathrm{TeV}$
}

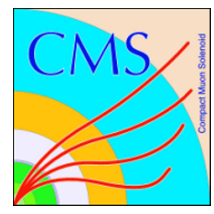

\section{The CMS collaboration}

E-mail: cms-publication-committee-chair@cern.ch

ABSTRACT: A search for standard model Higgs bosons $(\mathrm{H})$ produced with transverse momentum $\left(p_{\mathrm{T}}\right)$ greater than $450 \mathrm{GeV}$ and decaying to bottom quark-antiquark pairs $(\mathrm{b} \overline{\mathrm{b}})$ is performed using proton-proton collision data collected by the CMS experiment at the LHC at $\sqrt{s}=13 \mathrm{TeV}$. The data sample corresponds to an integrated luminosity of $137 \mathrm{fb}^{-1}$. The search is inclusive in the Higgs boson production mode. Highly Lorentz-boosted Higgs bosons decaying to $b \bar{b}$ are reconstructed as single large-radius jets, and are identified using jet substructure and a dedicated $\mathrm{b}$ tagging technique based on a deep neural network. The method is validated with $\mathrm{Z} \rightarrow \mathrm{b} \overline{\mathrm{b}}$ decays. For a Higgs boson mass of $125 \mathrm{GeV}$, an excess of events above the background assuming no Higgs boson production is observed with a local significance of 2.5 standard deviations $(\sigma)$, while the expectation is 0.7 . The corresponding signal strength and local significance with respect to the standard model expectation are $\mu_{\mathrm{H}}=3.7 \pm 1.2$ (stat) $)_{-0.7}^{+0.8}(\text { syst })_{-0.5}^{+0.8}$ (theo) and $1.9 \sigma$. Additionally, an unfolded differential cross section as a function of Higgs boson $p_{\mathrm{T}}$ for the gluon fusion production mode is presented, assuming the other production modes occur at the expected rates.

KEYworDs: Hadron-Hadron scattering (experiments), Higgs physics

ArXiv EPrint: 2006.13251 


\section{Contents}

1 Introduction 1

2 The CMS detector 2

3 Simulated samples $\quad 3$

4 Event reconstruction and selection 4

$\begin{array}{llr}5 & \text { Background estimation } & 7\end{array}$

$\begin{array}{llr}6 & \text { Systematic uncertainties } & 11\end{array}$

$\begin{array}{lll}7 & \text { Results } & 13\end{array}$

$\begin{array}{lll}8 & \text { Summary } & 19\end{array}$

$\begin{array}{lr}\text { The CMS collaboration } & 29\end{array}$

\section{Introduction}

The observation of a new boson consistent with the standard model (SM) Higgs boson $(\mathrm{H})$ and the subsequent measurements of its properties [1-3] have advanced the understanding of electroweak (EW) symmetry breaking and the origin of the mass of elementary particles [4-11]. The $\mathrm{H}$ boson has been observed at the CERN LHC in all of its main expected production modes and several decay modes, including decays to bottom quarkantiquark pairs $(b \bar{b})$ when produced in association with a $\mathrm{W}$ or $\mathrm{Z}$ boson $[12,13]$. Recently, there has been considerable interest in the measurement of Higgs bosons produced with high transverse momentum, $p_{\mathrm{T}}$, where measurements in the $\mathrm{H}(\mathrm{b} \overline{\mathrm{b}})$ decay channel have better sensitivity than traditional channels because of its large branching fraction, $\mathcal{B}(\mathrm{H} \rightarrow \mathrm{b} \overline{\mathrm{b}})=58.1 \%$ [14]. Advances in the identification of large-radius jets [15-19] resulting from massive color singlet particles with large transverse momentum and decaying to $b \bar{b}$ pairs have improved the sensitivity of this channel, as demonstrated by the CMS [20, 21] and ATLAS [22] Collaborations. The first search for high- $p_{\mathrm{T}} \mathrm{H}(\mathrm{b} \overline{\mathrm{b}})$ events by the CMS Collaboration [23] demonstrated the experimental sensitivity of this channel, with an expected significance of 0.7 standard deviations $(\sigma)$ based on a different theoretical expectation than the latest one used in this paper. Measurements of high- $p_{\mathrm{T}} \mathrm{H}(\mathrm{b} \overline{\mathrm{b}})$ events provide an alternative approach to study the top quark Yukawa coupling, complementary to associated $\mathrm{H}$ production with a top quark-antiquark pair $(\mathrm{t} \overline{\mathrm{t}} \mathrm{H})$, and may be sensitive to effects from physics beyond the SM [24-31]. At the highest $p_{\mathrm{T}}$, this measurement can resolve loop-induced contributions to the $\mathrm{ggH}$ process from new particles, such as a top quark partner, which would be described by an effective gg $\mathrm{H}$ vertex at low $p_{\mathrm{T}}$. 
This paper reports the results of an inclusive search for high- $p_{\mathrm{T}}$ Higgs bosons decaying to $\mathrm{b} \overline{\mathrm{b}}$ pairs in proton-proton $(\mathrm{pp})$ collisions at $\sqrt{s}=13 \mathrm{TeV}$. The data set, collected with the CMS detector at the LHC in 2016-2018, corresponds to an integrated luminosity of $137 \mathrm{fb}^{-1}$. The search is inclusive in the Higgs boson production mode. The highly Lorentz-boosted $\mathrm{H}(\mathrm{b} \overline{\mathrm{b}})$ candidates are reconstructed as single large-radius jets with the jet mass consistent with that of the observed Higgs boson [19]. The candidate jet is required to have $p_{\mathrm{T}}>450 \mathrm{GeV}$ to satisfy restrictive trigger requirements that suppress the large background from jets produced via the strong interaction, referred to as quantum chromodynamics (QCD) multijet events. To further distinguish the $\mathrm{H}$ candidates from the background, the jet is required to have a two-prong substructure, as well as displaced tracks and decay vertices consistent with the $\mathrm{H}(\mathrm{b} \overline{\mathrm{b}})$ signal, identified with a dedicated algorithm that detects the presence of $\mathrm{b}$ hadrons in the jet ( $\mathrm{b}$ tagging). The events are divided into six adjacent $p_{\mathrm{T}}$ categories. The background from QCD multijet production is difficult to model parametrically, and it is therefore estimated in data by relating the event yields in the signal region to those in a control region defined by inverting the $b$ tagging requirement, which is designed to have reduced correlation with jet mass and $p_{\mathrm{T}}$. The presence of the $\mathrm{W}$ and $\mathrm{Z}$ boson resonances in the jet mass distribution is used to constrain various systematic uncertainties and to validate the analysis. A separate control region is used to improve the modeling of the $t \bar{t}$ background. A simultaneous fit to the distributions of the jet mass in all $p_{\mathrm{T}}$ categories is performed to determine the normalizations and shapes of the jet mass distributions for the backgrounds and to extract the inclusive $\mathrm{H}(\mathrm{b} \overline{\mathrm{b}})$ signal strength with respect to the SM expectation. The differential cross section for the ggH Higgs boson $p_{\mathrm{T}}$ is also extracted under the assumption that $\mathrm{H}$ production through other modes occurs at the SM rate.

In contrast with the previous CMS result, the Higgs boson $p_{\mathrm{T}}$ spectrum from ggH production is modeled with the HJ-MiNLO generator [32-34], which includes effects of the finite top quark mass effects to higher order in QCD. The predicted cross section is compatible with the latest theoretical calculations [35, 36], and is smaller than that used previously [23]. Another major improvement is the development of a b tagging algorithm based on a deep neural network with better $\mathrm{H}(\mathrm{b} \overline{\mathrm{b}})$ signal efficiency.

This paper is organized as follows. A brief description of the CMS detector is given in section 2. Section 3 provides a summary of the various simulated samples used in the analysis. Section 4 describes the event reconstruction and selection criteria used to define the signal and control regions. The background estimation methods are detailed in section 5. Section 6 lists the sources of systematic uncertainty and their statistical treatment. Section 7 describes the statistical procedure used to derive the results, and reports the results in terms of signal strength modifiers and differential cross sections. Finally, the results are summarized in section 8.

\section{The CMS detector}

The central feature of the CMS apparatus is a superconducting solenoid of $6 \mathrm{~m}$ internal diameter, providing a magnetic field of $3.8 \mathrm{~T}$ inside its volume. Within the solenoid volume 
are a silicon pixel and strip tracker, a lead tungstate crystal electromagnetic calorimeter, and a brass and scintillator hadron calorimeter, each composed of a barrel and two endcap sections. Forward calorimeters extend the pseudorapidity $(\eta)$ coverage provided by the barrel and endcap detectors. Muons are detected in gas-ionization chambers embedded in the steel flux-return yoke outside the solenoid.

Events of interest are selected using a two-tiered trigger system [37]. The first level, composed of custom hardware processors, uses information from the calorimeters and muon detectors to select events at a rate of around $100 \mathrm{kHz}$ within a time interval of less than $4 \mu \mathrm{s}$. The second level, known as the high-level trigger, consists of a farm of processors running a version of the full event reconstruction software optimized for fast processing, and reduces the event rate to around $1 \mathrm{kHz}$ before data storage.

A more detailed description of the CMS detector, together with a definition of the coordinate system used and the relevant kinematic variables, can be found in ref. [38].

\section{Simulated samples}

Simulated samples of signal and background events are produced using various Monte Carlo (MC) event generators, with the CMS detector response modeled by GEANT4 [39].

For 2016 running conditions, the QCD multijet and Z+jets processes are modeled at leading order (LO) accuracy using the MADGRAPH5_aMC@NLO v2.2.2 generator [40]. The $\mathrm{W}+$ jets process is modeled at LO accuracy with MADGRAPH5_amC@NLO v2.3.3. The vector boson $(\mathrm{V})$ samples include decays of the bosons to all flavors of quarks, $\mathrm{V}(\mathrm{q} \overline{\mathrm{q}})$, and include up to 3 (4) extra partons at the matrix element level for $\mathrm{W}+$ jets ( $\mathrm{Z}+$ jets). Jets from the matrix element calculation and the parton shower description are matched using the MLM prescription [41]. The $t \overline{\mathrm{t}}$ and single top quark processes are modeled at nextto-LO (NLO) using POWHEg 2.0 [42-47]. Diboson processes are modeled at LO accuracy with PYTHIA 8.205 [48].

For 2017 and 2018 running conditions, the same configurations are used, but with newer generator versions. The QCD multijet and $\mathrm{V}+$ jets processes are modeled using MADGRAPH5_aMC@NLO v2.4.2, and the diboson processes are modeled with PYTHIA 8.226.

For all years, the cross sections for the $\mathrm{V}+$ jets samples are corrected as functions of boson $p_{\mathrm{T}}$ for higher-order QCD and EW effects. The QCD NLO corrections are derived using MADGRAPH5_aMC@NLO, simulating W and Z production with up to 2 additional partons and FXFx matching to the parton shower [49]. The EW NLO corrections are taken from theoretical calculations in refs. [50-53]. Additionally, the total cross sections for the diboson samples are corrected to next-to-NLO (NNLO) accuracy with the MCFM 7.0 program [54].

The $\mathrm{ggH}$ production process is simulated using the HJ-MiNLO [32, 33, 43, 55] event generator with mass $m_{\mathrm{H}}=125 \mathrm{GeV}$ and including finite top quark mass effects, following the recommendation in ref. [33]. Additionally, a sample of $\mathrm{ggH}$ events is generated with POWHEG [56] and corrected for the effects of the finite top quark mass using the same procedure as described in ref. [23], where the NLO to LO ratio of the $p_{\mathrm{T}}$ spectrum is approximated by expanding in powers of the inverse square of the top quark mass. The 
POWHEG generator is used to model Higgs boson production through vector boson fusion (VBF), VH associated production, and $\mathrm{t} \overline{\mathrm{t}} \mathrm{H}$ channels $[55,57,58]$. The $p_{\mathrm{T}}$ spectrum of the Higgs boson for the VBF production mode is re-weighted to account for next-to-NNLO corrections to the cross section $[59,60]$. These corrections have a negligible effect on the yield for this process for events with Higgs boson $p_{\mathrm{T}}>450 \mathrm{GeV}$.

For parton showering and hadronization, the POWHEG and MADGRAPH5_aMC@NLO samples are interfaced with PYTHIA 8.205 (8.230) for 2016 (2017 and 2018) running conditions. The PYTHIA parameters for the underlying event description are set to

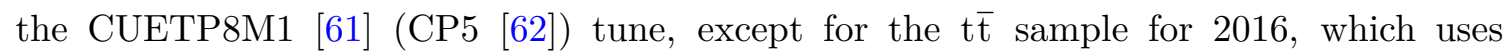
the CUETP8M2T4 tune [63]. For 2016 samples, the parton distribution function set NNPDF3.0 [64] is used, with the accuracy (LO or NLO) corresponding to that used in the matrix element calculations, while for 2017 and 2018 samples, NNPDF3.1 [65] at NNLO accuracy is used for all processes.

\section{Event reconstruction and selection}

Event reconstruction is based on a particle-flow algorithm [66], which aims to reconstruct and identify each individual particle with an optimized combination of information from the various elements of the CMS detector. The algorithm identifies each reconstructed particle as an electron, a muon, a photon, or a charged or neutral hadron. The missing transverse momentum vector is defined as the negative vector sum of the transverse momenta of all the particles identified in the event, and its magnitude is referred to as $p_{\mathrm{T}}^{\text {miss }}$. The candidate vertex with the largest value of summed physics-object $p_{\mathrm{T}}^{2}$ is taken to be the primary pp interaction vertex. The physics objects are the jets, clustered using the jet finding algorithm [67] with the tracks assigned to candidate vertices as inputs, and the associated missing transverse momentum, taken as the negative vector sum of the $p_{\mathrm{T}}$ of those jets.

Particles are clustered into jets using the anti- $k_{\mathrm{T}}$ algorithm with a distance parameter of 0.8 (AK8 jets) or 0.4 (AK4 jets). The larger radius of the AK8 jet better captures the decay products of the high- $p_{\mathrm{T}} \mathrm{H}(\mathrm{b} \overline{\mathrm{b}})$ signal. The clustering algorithms are implemented by the FASTJET package [68]. To mitigate the effect from the contributions of simultaneous pp collisions (pileup), the pileup per-particle identification algorithm [69, 70] assigns a weight to each particle prior to jet clustering based on the likelihood of the particle to originate from the hard scattering vertex. Further corrections are applied to the jet energy as a function of jet $\eta$ and $p_{\mathrm{T}}$ to bring the average measured response of jets to that of jets made directly from the generated particles before simulation of the detector response [71]. These corrections are derived separately for each data collection year. Jet identification criteria are applied to remove spurious jets associated with calorimeter noise as well as those associated with muon and electron candidates that are either misreconstructed or isolated. Specifically, jets are required to have neutral hadron and photon energy fractions less than 90\%, nonzero charged hadron energy fractions, muon energy fractions less than $80 \%$, and at least two constituent particles [72]. Additionally, AK8 jets are rejected if a photon with $p_{\mathrm{T}}>175 \mathrm{GeV}$ is reconstructed within the jet. 
A combination of several event selection criteria is used for the event trigger, all of which impose minimum thresholds on either the AK8 jet $p_{\mathrm{T}}$ or the event $H_{\mathrm{T}}$, defined as the scalar $p_{\mathrm{T}}$ sum of all jets in the event with $|\eta|<3.0$. For AK8 jets used in the trigger selection, a minimum threshold is also imposed on the trimmed jet mass [73], where remnants of soft radiation are removed before computing the mass, which allows the $H_{\mathrm{T}}$ or $p_{\mathrm{T}}$ thresholds to be reduced while maintaining manageable trigger rates. The trigger selection efficiency is greater than $95 \%$ for events with at least one AK8 jet with $|\eta|<2.5$, mass greater than $47 \mathrm{GeV}$ and $p_{\mathrm{T}}>450(525,500) \mathrm{GeV}$ for $2016(2017,2018)$ data.

To reduce backgrounds from SM EW processes, events are vetoed if they contain isolated electrons, isolated muons, or hadronically decaying $\tau$ leptons with $p_{\mathrm{T}}>10,10$, or $18 \mathrm{GeV}$ and $|\eta|<2.5,2.4$, or 2.3 , respectively. For electrons and muons, an isolation variable is calculated as the pileup-corrected $p_{\mathrm{T}}$ sum of the charged hadrons and neutral particles surrounding the lepton divided by the lepton $p_{\mathrm{T}}$. For charged particles, only those associated with the primary vertex are considered in the isolation variable. For neutral particles, the pileup correction consists of subtracting the energy deposited in the isolation cone by charged hadrons not associated with the primary vertex, multiplied by a factor of 0.5. This factor corresponds approximately to the ratio of neutral to charged hadron production in pileup interactions [74]. The isolation variable for electrons and muons is required to be less than 15 or $25 \%$, respectively, depending on $\eta[75,76]$.

For each event, the leading AK8 jet in $p_{\mathrm{T}}$ is selected to be the $\mathrm{H}(\mathrm{b} \overline{\mathrm{b}})$ candidate, which is around $60 \%$ efficient for the $\mathrm{ggH}$ production mode. Alternative $\mathrm{H}(\mathrm{b} \overline{\mathrm{b}})$ candidate jet selection criteria were considered, but were not found to improve the sensitivity. The AK8 jet is required to have $|\eta|<2.5$. To reduce the top quark contamination, events are vetoed if they have $p_{\mathrm{T}}^{\text {miss }}>140 \mathrm{GeV}$, or if they contain a b-tagged [20] AK4 jet with $p_{\mathrm{T}}>30 \mathrm{GeV}$ located in the opposite hemisphere from the leading AK8 jet $(\Delta \phi(\mathrm{AK} 4, \mathrm{AK} 8)>\pi / 2)$. The chosen threshold for the AK4 jet b-tagging algorithm corresponds to a $1 \%$ probability to misidentify a jet arising from a light flavor quark or gluon and a $77 \%$ probability to correctly identify a jet arising from a b quark in 2017 detector conditions. Approximately $60 \%$ of $\mathrm{t} \overline{\mathrm{t}}$ events are rejected by this selection.

The soft-drop (SD) algorithm [77] with angular exponent $\beta=0$ and soft radiation fraction $z=0.1$ is applied to the Higgs boson jet candidate to remove soft and wideangle radiation. The parameter $\beta$ controls the grooming profile as a function of subjet separation; for $\beta=0$, the algorithm is independent of subjet separation, and is equivalent to the modified mass-drop tagger [78]. The resulting SD jet mass, $m_{\mathrm{SD}}$, is strongly reduced for background QCD multijet events, where large jet masses arise from wide-angle gluon radiation. Conversely, the algorithm preserves the mass of jets from heavy boson decays. Corrections to the $m_{\mathrm{SD}}$ values from simulation are derived from a comparison of simulated and measured samples in a region enriched with merged $\mathrm{W}(\mathrm{q} \overline{\mathrm{q}})$ decays from $\mathrm{t} \overline{\mathrm{t}}$ events [72]. The $m_{\mathrm{SD}}$ corrections remove a residual dependence on the jet $p_{\mathrm{T}}$, and match the simulated jet mass scale and resolution to those observed in data.

The resulting $m_{\mathrm{SD}}$ distributions are binned from 47 to $201 \mathrm{GeV}$ with a bin width of $7 \mathrm{GeV}$. The lower bound is sufficiently above the trigger threshold to be insensitive to differences between the online and offline mass calculations, and the bin width corresponds to 
the $m_{\mathrm{SD}}$ resolution near the $\mathrm{V}$ resonances. The dimensionless mass scale variable for $\mathrm{QCD}$ multijet jets, $\rho\left(m_{\mathrm{SD}}, p_{\mathrm{T}}\right)=2 \ln \left(m_{\mathrm{SD}} / p_{\mathrm{T}}\right)[78,79]$, is used to characterize the correlation between the jet $\mathrm{b}$ tagging discriminator, jet mass, and jet $p_{\mathrm{T}}$. Its distribution is roughly invariant in different ranges of jet $p_{\mathrm{T}}$. For each $p_{\mathrm{T}}$ category, only those $m_{\mathrm{SD}}$ bins that satisfy

$$
\rho\left(0.5 m_{\mathrm{SD}}^{\mathrm{lo}}+0.5 m_{\mathrm{SD}}^{\mathrm{up}}, 0.7 p_{\mathrm{T}}^{\mathrm{lo}}+0.3 p_{\mathrm{T}}^{\mathrm{up}}\right)<-2.1
$$

are considered, where $m_{\mathrm{SD}}^{\mathrm{up}}\left(p_{\mathrm{T}}^{\mathrm{up}}\right)$ is the upper $m_{\mathrm{SD}}\left(p_{\mathrm{T}}\right)$ bound and $m_{\mathrm{SD}}^{\text {lo }}\left(p_{\mathrm{T}}^{\text {lo }}\right)$ is the lower $m_{\mathrm{SD}}\left(p_{\mathrm{T}}\right)$ bound. In this restriction, the lower $p_{\mathrm{T}}$ bound is weighted more heavily because of the steeply falling QCD multijet $p_{\mathrm{T}}$ distribution. This upper bound on $\rho$ is imposed to avoid instabilities at the edges of the distribution due to finite cone limitations from the jet clustering. This requirement is about $98 \%$ efficient for the $\mathrm{H}(\mathrm{b} \overline{\mathrm{b}})$ signal.

The $N_{2}^{1}$ variable [80] is used to determine how consistent a jet is with having a twoprong substructure. It is based on a ratio of 2-point $\left({ }_{1} e_{2}\right)$ and 3-point $\left.{ }_{2} e_{3}\right)$ generalized energy correlation functions [81]:

$$
\begin{aligned}
{ }_{1} e_{2} & =\sum_{1 \leq i<j \leq n} z_{i} z_{j} \Delta R_{i j}, \\
{ }_{2} e_{3} & =\sum_{1 \leq i<j<k \leq n} z_{i} z_{j} z_{k} \min \left\{\Delta R_{i j} \Delta R_{i k}, \Delta R_{i j} \Delta R_{j k}, \Delta R_{i k} \Delta R_{j k}\right\},
\end{aligned}
$$

where $z_{i}$ represents the energy fraction of the constituent $i$ in the jet, and $\Delta R_{i j}$ is the angular separation between constituents $i$ and $j$. These generalized energy correlation functions ${ }_{v} e_{n}$ are sensitive to correlations of $v$ pairwise angles among $n$ jet constituents [80]. For a two-prong structure, signal jets have a stronger 2-point correlation than a 3-point one. The discriminant variable $N_{2}^{1}$ is defined as

$$
N_{2}^{1}=\frac{{ }_{2} e_{3}}{\left({ }_{1} e_{2}\right)^{2}} .
$$

The calculation of $N_{2}^{1}$ is based on the jet constituents after application of the SD grooming algorithm to the jet. It provides excellent discrimination between two-prong signal jets and QCD background jets. However, imposing requirements on $N_{2}^{1}$, or other similar variables, distorts the jet mass distributions differently depending on the jet $p_{\mathrm{T}}$ [82]. To minimize this distortion, a transformation is applied to $N_{2}^{1}$ following the designed decorrelated tagger technique [79], reducing its correlation with $\rho$ and $p_{\mathrm{T}}$ in multijet events. The transformed variable is defined as $N_{2}^{1, \mathrm{DDT}} \equiv N_{2}^{1}-X_{(26 \%)}$, where $X_{(26 \%)}$ is the value corresponding to the 26th percentile of the $N_{2}^{1}$ distribution in simulated QCD events, as a function of $\rho$ and $p_{\mathrm{T}}$. The transformation is derived in bins of $\rho$ and $p_{\mathrm{T}}$. This ensures that the selection $N_{2}^{1, \mathrm{DDT}}<0$ yields a constant background efficiency for QCD events across the $\rho$ and $p_{\mathrm{T}}$ range considered in this search. The chosen efficiency of $26 \%$ maximizes the signal sensitivity.

Jets likely to originate from the merging of the fragmentation products of two $b$ quarks are selected using an algorithm based on a deep neural network, composed of multiple layers between input and output, referred to here as the deep double-b tagger (DDBT) [20, 21]. The algorithm takes as inputs several high-level observables that characterize the 
distinct properties of $\mathrm{b}$ hadrons and their momentum directions in relation to the two subjet candidate axes, as well as low-level track and vertex observables. Events where the selected AK8 jet is double-b tagged constitute the "passing," or signal, region, while events failing the DDBT form the "failing" region, which is used to estimate the QCD multijet background in the signal region. Specifically, an AK8 jet is considered double-b tagged if its DDBT discriminator value exceeds a threshold corresponding approximately to a $1 \%$ misidentification probability for QCD jets. This threshold corresponds to a $54 \%$ efficiency for reconstructed scalar boson resonances with variable masses decaying to $\mathrm{b} \overline{\mathrm{b}}$ in the range $40<m_{\mathrm{SD}}<200 \mathrm{GeV}$ and $450<p_{\mathrm{T}}<1200 \mathrm{GeV}$ in simulation corresponding to the detector conditions in 2017. The performance of the DDBT algorithm for 2018 detector conditions is approximately the same, while the performance for 2016 ones is slightly worse ( $45 \%$ efficiency for $\mathrm{b} \overline{\mathrm{b}}$ resonances in the same $m_{\mathrm{SD}}$ and $p_{\mathrm{T}}$ range and for the same misidentification probability) because the CMS pixel tracker was upgraded between 2016 and 2017 [83]. Compared to the previous double-b tagger (DBT) algorithm [20] used in a prior CMS result [23], the DDBT improves the $b \bar{b}$ tagging efficiency by a factor of about 1.6 for the same detector conditions and QCD misidentification probability. For SM $\mathrm{ggH}$ production specifically, the tagging efficiency is approximately $60 \%$, an improvement over the previous algorithm by a factor of about 1.3. Figure 1 shows the performance curves of misidentification probability for QCD jets versus the identification probability for $b \bar{b}$ resonance jets for the previous DBT algorithm and the DDBT algorithm in simulation corresponding to 2017 detector conditions.

After all selections are applied, the Higgs boson candidate jet is categorized into the DDBT passing or failing region, each with $22 m_{\mathrm{SD}}$ bins evenly dividing the range 47$201 \mathrm{GeV}$, and split further into six jet $p_{\mathrm{T}}$ categories with bin boundaries of 450, 500, 550, $600,675,800$, and $1200 \mathrm{GeV}$. The upper $p_{\mathrm{T}}$ bound of $1200 \mathrm{GeV}$ does not have a significant impact on the sensitivity and excludes a region where the QCD multijet background is difficult to model. The remaining $p_{\mathrm{T}}$ binning is optimized for best signal significance, and the upper $m_{\mathrm{SD}}$ bound is due to the requirements imposed on the jet $\rho$. Specifically, bins that do not satisfy eq. (4.1) are removed, resulting in a total of 124 bins each for the passing and failing regions. Namely, the upper $m_{\mathrm{SD}}$ bound for the first two $p_{\mathrm{T}}$ categories are $166 \mathrm{GeV}$ and $180 \mathrm{GeV}$, respectively. For the Higgs boson signal processes in the DDBT passing region, the dominant production mode is $\mathrm{ggH}(56 \%)$, followed by $\mathrm{VBF}(26 \%), \mathrm{VH}$ (13\%), and $\mathrm{t} \overline{\mathrm{t}} \mathrm{H}(5 \%)$.

\section{Background estimation}

The dominant background in the signal region is QCD multijet production. The $\mathrm{V}+$ jets processes are significant resonant backgrounds. The $t \bar{t}$ process constitutes a significant nonresonant background across the $m_{\mathrm{SD}}$ spectrum. Other EW processes, including diboson, triboson, and $t \overline{\mathrm{t}} \mathrm{V}$, are estimated from simulation and found to be negligible.

The $\mathrm{V}+$ jets background is modeled using simulation. Their overall contribution is less than $6 \%$ of the total background in the DDBT passing region. The normalizations and shapes of the simulated $\mathrm{V}+$ jets background are corrected for NLO QCD and EW effects. 


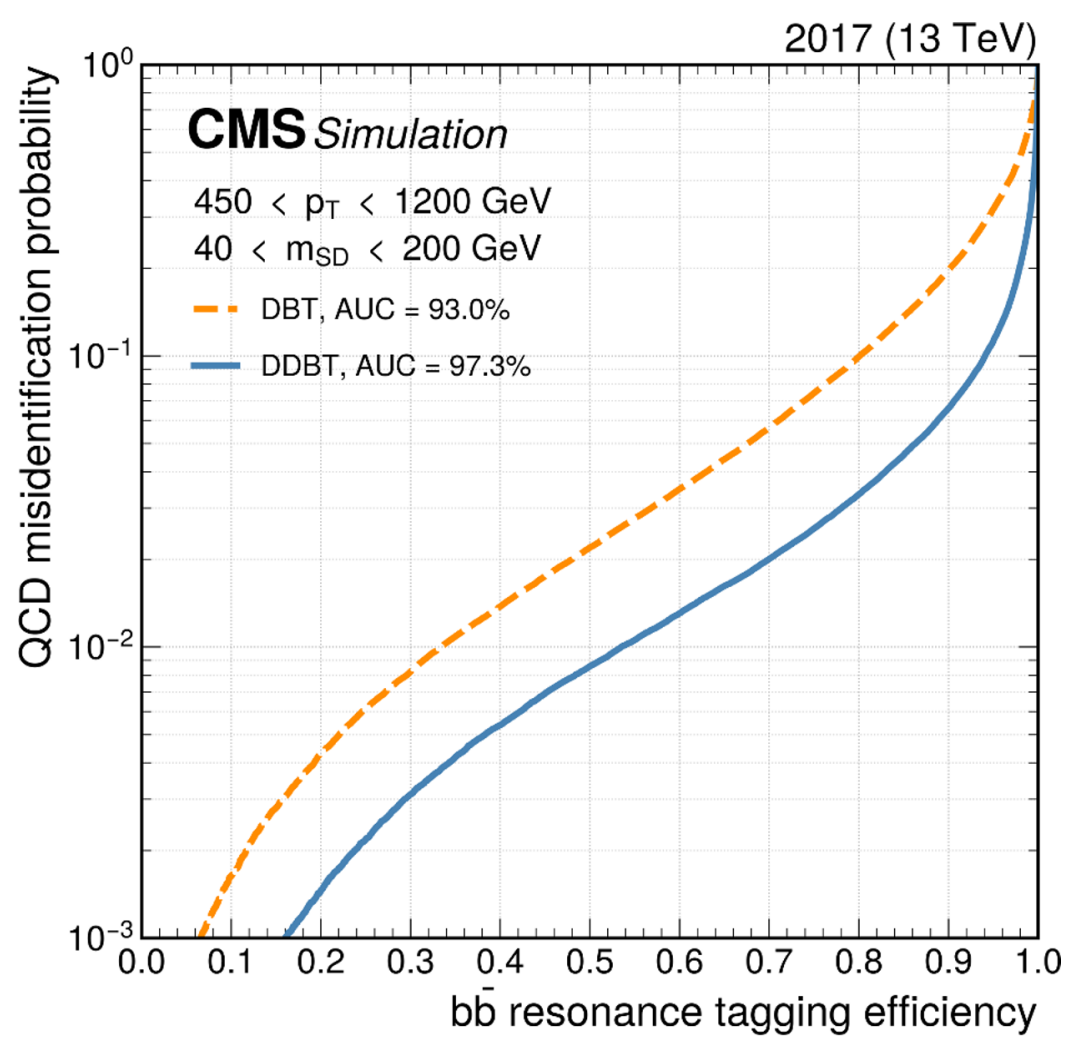

Figure 1. The performance curves of misidentification probability for jets originating from QCD multijet production versus the identification probability for $b \bar{b}$ resonance jets for the DBT (orange dashed line) used in a prior CMS result and the DDBT (blue solid line). The $\mathrm{b} \overline{\mathrm{b}}$ resonances are generated with variable masses in the range $15-250 \mathrm{GeV}$. The curves are evaluated with simulation corresponding to the detector conditions in 2017. Jets are required to have $p_{\mathrm{T}}$ in the range 450$1200 \mathrm{GeV}$ and $m_{\mathrm{SD}}$ in the range $40-200 \mathrm{GeV}$. The area under the curve (AUC) is reported as a performance metric for both algorithms.

The contribution of $\mathrm{t} \overline{\mathrm{t}}$ production to the total background is obtained from simulation, where the normalization and DDBT efficiency are corrected with scale factors derived

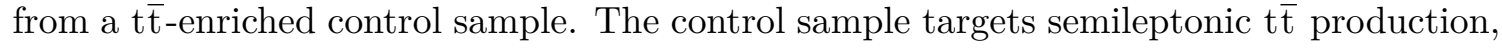
consisting of events with an energetic muon with $p_{\mathrm{T}}>55 \mathrm{GeV}$ and $|\eta|<2.1$, a leading AK8 jet with $p_{\mathrm{T}}>400 \mathrm{GeV}$, and an additional b-tagged AK4 jet that is separated from the leading AK8 jet by $\Delta R>0.8$. The AK8 jet with the highest $p_{\mathrm{T}}$ is taken to be the candidate jet. Using the same candidate jet requirements that define the signal selection, DDBT passing and failing regions are constructed in both data and simulation. Due to the relatively low event count in the control sample, the inclusive event counts for $47<$ $m_{\mathrm{SD}}<201 \mathrm{GeV}$ and $p_{\mathrm{T}}>400 \mathrm{GeV}$ are used, totalling 438 (6301) events in the data passing (failing) region. The fraction of $t \bar{t}$ background relative to the total background expected in this control sample is $72 \%$. Both the absolute normalization and DDBT efficiency of the $t \bar{t}$ contribution are allowed to vary without constraint from the simulation expectation, but are forced to vary identically in the $t \bar{t}$ control region and the signal region in the simultaneous fit, thus constraining the background expectation and DDBT mistag probability for this 
process. The net contribution is about $8 \%$ of the total background in the $110<m_{\mathrm{SD}}<$ $131 \mathrm{GeV}$ range of the DDBT passing region.

The main background in the DDBT passing region, QCD multijet production, has a jet mass shape that depends on $p_{\mathrm{T}}$ and is difficult to model parametrically. Therefore, we estimate it using the background-enriched failing region, i.e., events failing the DDBT selection, together with a "pass-fail ratio" function, $R_{\mathrm{p} / \mathrm{f}}$. Ideally, $R_{\mathrm{p} / \mathrm{f}}$ would be constant as a function of jet mass and $p_{\mathrm{T}}$, as the DDBT discriminator is designed to be uncorrelated from both variables: the training procedure incorporates a penalty term to the loss function for differences in the jet mass distribution between the passing and failing events, and the training samples are weighted such that the loss function is independent of jet $p_{\mathrm{T}}$. Nonetheless, the DDBT exhibits some anticorrelation at high tagger discriminator values and low jet mass, i.e., the mass distributions are different in the passing and failing regions. Additionally, residual differences in $R_{\mathrm{p} / \mathrm{f}}$ may arise from discrepancies in tagger performance between data and simulation. To account for both effects, $R_{\mathrm{p} / \mathrm{f}}$ is separated into two components: an expected pass-fail ratio is taken from simulated QCD multijet events by fitting a two-dimensional second-order Bernstein polynomial [84] in $\rho$ and $p_{\mathrm{T}}, \epsilon^{\mathrm{QCD}}\left(\rho, p_{\mathrm{T}}\right)$, to the distributions in simulation; and a data residual correction is parametrized using a Bernstein polynomial in $\rho$ and $p_{\mathrm{T}}$. The complete pass-fail ratio in data is given by the product of these two factors,

$$
R_{\mathrm{p} / \mathrm{f}}\left(\rho, p_{\mathrm{T}}\right)=\sum_{k=0}^{n_{\rho}} \sum_{\ell=0}^{n_{p_{\mathrm{T}}}} a_{k, \ell} b_{k, n_{\rho}}(\rho) b_{\ell, n_{p_{\mathrm{T}}}}\left(p_{\mathrm{T}}\right) \epsilon^{\mathrm{QCD}}\left(\rho, p_{\mathrm{T}}\right),
$$

where $n_{\rho}$ is the degree of the polynomial in $\rho, n_{p_{\mathrm{T}}}$ is the degree of the polynomial in $p_{\mathrm{T}}$, $a_{k, \ell}$ is a Bernstein coefficient, and

$$
b_{\nu, n}(x)=\left(\begin{array}{l}
n \\
\nu
\end{array}\right) x^{\nu}(1-x)^{n-\nu}
$$

is a Bernstein basis polynomial of degree $n$.

The pass-fail ratio $R_{\mathrm{p} / \mathrm{f}}$ is determined from a simultaneous binned fit to the $m_{\mathrm{SD}}$ data distributions in the DDBT passing and failing regions across the whole jet mass and $p_{\mathrm{T}}$ range, accounting for the contributions from signal and non-QCD backgrounds. In this fit, the coefficients $a_{k, \ell}$ (data correction) are fitted with no external constraints, while the $\epsilon^{\mathrm{QCD}}$ coefficients and their associated uncertainties are taken from the separate fit to the QCD simulation. The $p_{\mathrm{T}}$ bin widths, which vary from 50 to $400 \mathrm{GeV}$, are chosen to provide enough data points to constrain the shape of $R_{\mathrm{p} / \mathrm{f}}$. To determine the minimum degree of polynomial necessary to fit the data, a Fisher $F$-test [85] is performed. As the magnitude of data-to-simulation discrepancies can vary among the data samples and their corresponding simulation samples, an $F$-test is performed independently for each of the three data taking years. For the 2016 data sample, it is found that a polynomial of order $\left(n_{\rho}, n_{p_{\mathrm{T}}}\right)=(2,1)$ is needed to provide a sufficient goodness of fit with respect to increased orders $(p>0.05)$, while for 2017 and 2018 data, a residual polynomial of order $\left(n_{\rho}, n_{p_{\mathrm{T}}}\right)=(1,1)$ is found to be sufficient. 


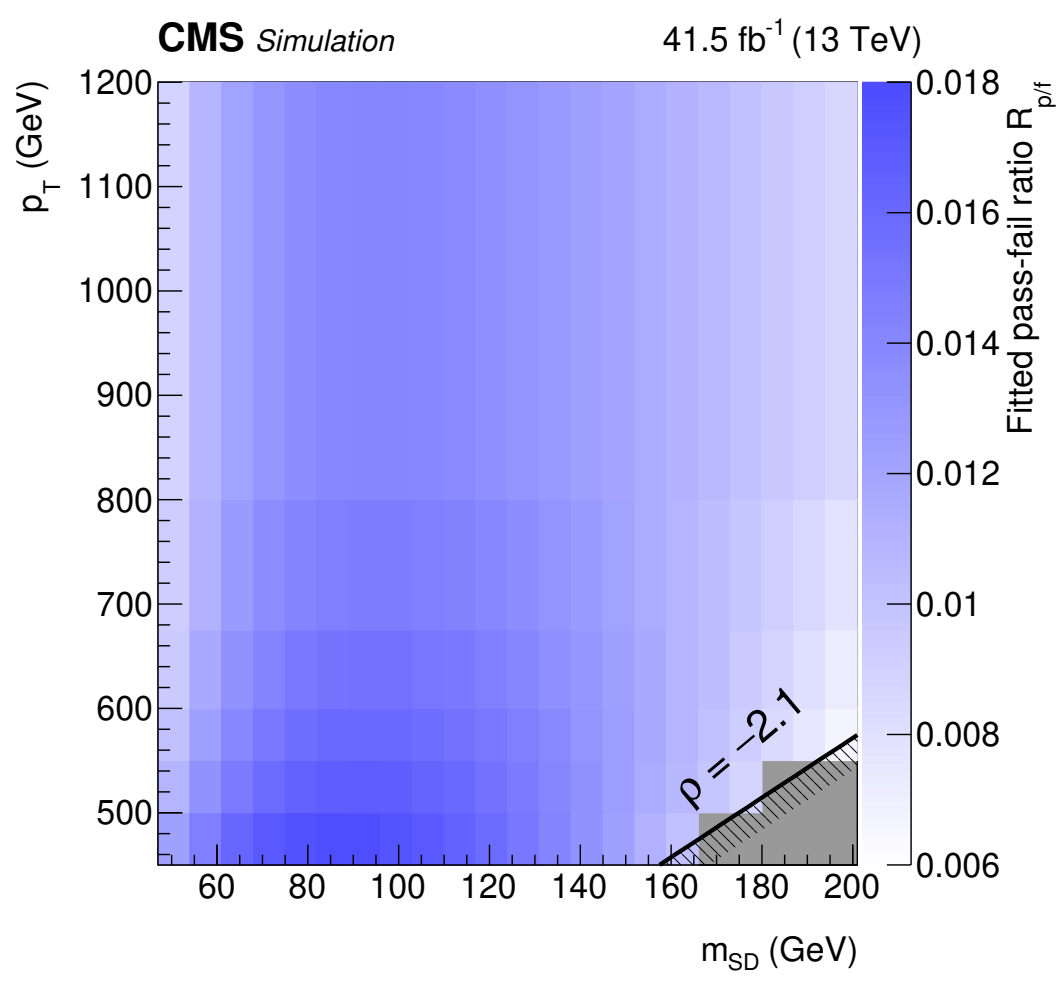

Figure 2. The fitted pass-fail ratio $R_{\mathrm{p} / \mathrm{f}}$ as a function of jet $p_{\mathrm{T}}$ and $m_{\mathrm{SD}}$ for data collected in 2017 . The ratio relates the QCD multijet event yield in the DDBT passing region to that of the failing region. The binning corresponds to the $22 m_{\mathrm{SD}}$ bins and $6 p_{\mathrm{T}}$ categories used in the statistical analysis. The lower-right bins filled in gray fall outside of the $\rho$ acceptance.

The 2017 fitted pass-fail ratio $R_{\mathrm{p} / \mathrm{f}}$ as a function of $m_{\mathrm{SD}}$ and $p_{\mathrm{T}}$ under the signal-plusbackground hypothesis is shown in figure 2 . In the absence of correlations between $m_{\mathrm{SD}}$, $p_{\mathrm{T}}$, and the DDBT efficiency, the ratio would be approximately 0.01 . The majority of the difference from 0.01 is a result of the expected pass-fail ratio, which ranges from 0.007 to 0.018 , while the data residual correction ranges from 0.86 to 1.05 . The other data taking periods are similar. As discussed in section 6, the components of the pass-fail ratio are among the largest sources of uncertainty in the analysis.

As the QCD background estimate relies solely on the properties of the $\mathrm{H}(\mathrm{b} \overline{\mathrm{b}})$ candidate jet, $\mathrm{V}+$ jets proceses in which the candidate jet does not arise from a vector boson decay are included in this estimate, and therefore are removed from the predicted yields of those processes.

In order to validate the background estimation method and associated systematic uncertainties, bias studies are performed using an alternative functional form for the pass-fail ratio in the background model. Pseudo-experiment data sets are generated assuming the alternative background model, with the injection of signal events for a range of hypothetical signal strength values of between 0 and 5 times the SM expectation, and then fit with the nominal signal-plus-background model. No significant bias in the fitted signal strength is observed; specifically, the means of the differences between the fitted and in- 
jected signal strengths divided by the fitted uncertainty are found to be less than $15 \%$. Therefore, no additional systematic uncertainty is assigned for this potential bias from the background modeling.

\section{Systematic uncertainties}

The systematic uncertainties associated with the jet mass scale, the jet mass resolution, and the $N_{2}^{1, \mathrm{DDT}}$ selection efficiency are correlated among the $\mathrm{W}, \mathrm{Z}$, and $\mathrm{H}(\mathrm{b} \overline{\mathrm{b}})$ processes. These uncertainties are estimated in data using an independent sample of merged $\mathrm{W}$ boson jets in semileptonic t $\overline{\mathrm{t}}$ events, where the hadronically decaying $\mathrm{W}$ boson is reconstructed as a single AK8 jet.

For this sample, data events are required to have an energetic muon with $p_{\mathrm{T}}>100 \mathrm{GeV}$ and $|\eta|<2.1, p_{\mathrm{T}}^{\text {miss }}>80 \mathrm{GeV}$, a high- $p_{\mathrm{T}}$ AK8 jet with $p_{\mathrm{T}}>200 \mathrm{GeV}$, and an additional b-tagged AK4 jet separated from the AK8 jet by $\Delta R>0.8$ with $p_{\mathrm{T}}>30 \mathrm{GeV}$. Using the same $N_{2}^{1, \mathrm{DDT}}$ requirement applied in the signal regions, we define two samples, one with events that pass and one with events that fail the $N_{2}^{1, \mathrm{DDT}}$ selection, for merged $\mathrm{W}$ boson jets in data and simulation. A simultaneous fit to the two samples in $m_{\mathrm{SD}}$ is performed in order to extract the selection efficiency of a merged $\mathrm{W}$ jet in simulation and in data. The data-to-simulation scale factors for the $N_{2}^{1, \mathrm{DDT}}$ selection efficiency are measured separately for the three data taking periods, as listed in table 1 .

The jet mass scale and jet mass resolution data-to-simulation scale factors are extracted from the same fit, and are also shown in table 1 . As the semileptonic t $\bar{t}$ sample does not contain a large population of very energetic jets, an additional systematic uncertainty is included to account for the extrapolation to very high $p_{\mathrm{T}}$ jets. This additional uncertainty is estimated to be $0.5 \%$ per $100 \mathrm{GeV}$, based on a study of fitting the $m_{\mathrm{SD}}$ distributions of merged top quark jets in different $p_{\mathrm{T}}$ ranges above $350 \mathrm{GeV}$ [86]. In total, the jet mass scale uncertainty increases with jet $p_{\mathrm{T}}$, ranging from $1.2 \%$ at $450 \mathrm{GeV}$ to $2.1 \%$ at $800 \mathrm{GeV}$. While the jet mass scale and resolution among the different years of data collection are similar, their data-to-simulation scale factors and uncertainties vary because of the different generator tunes used in the simulations.

The uncertainty on the efficiency of the DDBT is estimated using data and simulation samples enriched in $b \bar{b}$ pairs from gluon splitting [20]. The gluon splitting samples require that both subjets of an AK8 jet contain a muon, targeting semileptonic decays of the $\mathrm{b}$ hadrons. The method is based on yields extracted from fits to the distributions of the jet probability tagger $[20,87]$ discriminant, which uses the signed impact parameter significance of the tracks associated with the jet to obtain a likelihood for the jet to originate from the primary vertex.

Given that the DDBT efficiencies could differ between $b \bar{b}$ jets from gluon splitting and from color-singlet $\mathrm{Z}$ or Higgs boson decays, the efficiencies extracted from the gluon splitting samples are used only to estimate the uncertainty on the DDBT efficiency, and are not used to correct the efficiency. The applied DDBT data-to-simulation scale factor is included in the signal extraction fit as a constrained nuisance parameter, with a nominal value of unity and an uncertainty equal to the difference between the DDBT data-to- 


\begin{tabular}{|cccccc|}
\hline $\begin{array}{c}\text { Data } \\
\text { period }\end{array}$ & $\begin{array}{c}\text { Integrated } \\
\text { luminosity }\left(\mathrm{fb}^{-1}\right)\end{array}$ & Jet mass scale & Jet mass resolution & $N_{2}^{1, \text { DDT }}$ selection & $\begin{array}{c}\text { DDBT selection } \\
(\mathrm{g} \rightarrow \mathrm{b} \overline{\mathrm{b}})\end{array}$ \\
\hline 2016 & 35.9 & $1.000 \pm 0.012$ & $1.084 \pm 0.091$ & $0.993 \pm 0.043$ & $1.00 \pm 0.23$ \\
2017 & 41.5 & $0.987 \pm 0.012$ & $0.905 \pm 0.048$ & $0.924 \pm 0.018$ & $1.00 \pm 0.32$ \\
2018 & 59.2 & $0.970 \pm 0.012$ & $0.908 \pm 0.014$ & $0.953 \pm 0.016$ & $1.00 \pm 0.30$ \\
\hline
\end{tabular}

Table 1. Summary of applied data-to-simulation scale factors for the jet mass scale, jet mass resolution, $N_{2}^{1, D D T}$ selection, and DDBT selection for different data taking periods.

simulation scale factor and unity, as shown in table 1. The scale factor is further constrained via the observed $\mathrm{Z}$ boson yield in the passing and failing regions. This strategy differs from that of the previous CMS analysis [23], resulting in an increase in the post-fit systematic uncertainty of the tagger efficiency from $4 \%$ to about $14 \%$.

The scale factors described above determine the initial distributions of the jet mass for the $\mathrm{W}(\mathrm{q} \overline{\mathrm{q}}), \mathrm{Z}(\mathrm{q} \overline{\mathrm{q}})$, and $\mathrm{H}(\mathrm{b} \overline{\mathrm{b}})$ processes. In the fit to data, the jet mass scales and resolutions are treated as constrained nuisance parameters with nominal values and uncertainties as shown in table 1, and are further constrained by the presence of the $\mathrm{V}$ resonances in the jet mass distribution. A single nuisance parameter per year is considered for the $N_{2}^{1, \text { DDT }}$ selection efficiency uncertainty. Alternative configurations in which multiple nuisance parameters are considered for the $N_{2}^{1, \text { DDT }}$ selection efficiency uncertainty in order to account for a potential mass or $p_{\mathrm{T}}$ dependence were found to have no impact on the analysis results.

The uncertainty associated with the choice of QCD renormalization and factorization scales in the modeling of $\mathrm{ggH}$ production is propagated to the total expected yield of the $\mathrm{ggH}$ signal via varying each factor by one-half or two around the nominal value and finding the envelope of all combinations of such variations, except those where one scale is multiplied by 0.5 and the other is multiplied by $2[88,89]$. This results in a $30 \%$ uncertainty for the POWHEG sample with $p_{\mathrm{T}}$ reweighting [23] and a $20 \%$ uncertainty for the HJ-MiNLO sample. These variations account for the effect on both the inclusive cross section and acceptance. An additional uncertainty is considered for the reweighted POWHEG sample, in which the shape of the ggH Higgs boson $p_{\mathrm{T}}$ distribution is allowed to vary by a linear function of the Higgs boson $p_{\mathrm{T}}$ that changes the relative yield at $1.2 \mathrm{TeV}$ by $\pm 30 \%$ for a $1 \sigma$ effect, without changing the overall yield. Uncertainties related to finite top quark mass effects are estimated in ref. [36], and are found to be subdominant to the scale uncertainties for the HJ-MiNLO sample. For the V $(q \bar{q})$ yield, two nuisance parameters account for potential $p_{\mathrm{T}}$-dependent deviations due to missing higher-order corrections, where one is $10 \%$ in magnitude on the total yield, and the other increases from 0 to $7 \%$ versus $p_{\mathrm{T}}[50,51,90-94]$. An additional systematic uncertainty of 2 to $6 \%$, depending on $p_{\mathrm{T}}$, is included to account for potential differences between the higher-order corrections to the $\mathrm{W}$ and $\mathrm{Z}$ cross sections (EW W/Z decorrelation) [90].

Finally, systematic uncertainties are applied to the $\mathrm{W}(\mathrm{q} \overline{\mathrm{q}}), \mathrm{Z}(\mathrm{q} \overline{\mathrm{q}})$, t $\overline{\mathrm{t}}$, and $\mathrm{H}(\mathrm{b} \overline{\mathrm{b}})$ yields to account for the uncertainties due to the jet energy scale and resolution [95] and the limited simulation sample sizes. The effect of limited QCD simulation sample size on the separate fit to determine the expected pass-fail ratio $\epsilon^{\mathrm{QCD}}\left(\rho, p_{\mathrm{T}}\right)$ is also included. Other 


\begin{tabular}{|c|c|c|}
\hline Uncertainty source & \multicolumn{2}{|c|}{$\Delta \mu_{\mathrm{H}}$} \\
\hline Statistical & +1.2 & -1.2 \\
\hline Signal extraction & +0.9 & -0.8 \\
\hline QCD pass-fail ratio (data correction) & +0.8 & -0.7 \\
\hline $\mathrm{t} \overline{\mathrm{t}}$ normalization and misidentification & +0.4 & -0.4 \\
\hline Systematic & +0.8 & -0.7 \\
\hline QCD pass-fail ratio (simulation) & +0.6 & -0.6 \\
\hline DDBT efficiency & +0.3 & -0.1 \\
\hline Jet mass scale and resolution & +0.3 & -0.3 \\
\hline Jet energy scale and resolution & +0.1 & -0.1 \\
\hline Simulated sample size & +0.2 & -0.1 \\
\hline Other experimental uncertainties & +0.1 & -0.1 \\
\hline Theoretical & +0.8 & -0.5 \\
\hline $\mathrm{V}+$ jets modeling & +0.6 & -0.4 \\
\hline H modeling & +0.5 & -0.3 \\
\hline Total & +1.6 & -1.5 \\
\hline
\end{tabular}

Table 2. Major sources of uncertainty in the measurement of the signal strength $\mu_{\mathrm{H}}$ based on the HJ-MiNLO prediction, and their observed impact $\left(\Delta \mu_{\mathrm{H}}\right)$ from a fit to the combined data set. Decompositions of the statistical, systematic, and theoretical components of the total uncertainty are specified. The impact of each uncertainty is evaluated by computing the uncertainty excluding that source and subtracting it in quadrature from the total uncertainty. The sum in quadrature for each source does not in general equal the total uncertainty of each component because of correlations in the combined fit between nuisance parameters corresponding to different sources.

experimental uncertainties, including those related to the determination of the integrated luminosity [96], variations in the amount of pileup, modeling of the trigger acceptance, and the isolation and identification of leptons are also considered. Table 2 lists the major sources of uncertainty and their observed impact on the Higgs boson signal strength $\mu_{\mathrm{H}}$, defined as the ratio of the measured to the SM expected $\mathrm{H}(\mathrm{b} \overline{\mathrm{b}})$ production, in the combined fit. One of the largest sources of statistical uncertainty is the data residual correction to the pass-fail ratio $R_{\mathrm{p} / \mathrm{f}}$, while the largest source of systematic uncertainty is the expected pass-fail ratio $\epsilon^{\mathrm{QCD}}$, which is initially estimated from simulation and further constrained by the data. Overall, the $\mu_{\mathrm{H}}$ measurement is limited by statistical sources of uncertainty.

\section{Results}

A binned maximum likelihood fit to the observed $m_{\mathrm{SD}}$ distributions is performed using the sum of the signal and background contributions. The fit is performed simultaneously in the DDBT passing and failing regions of the six $p_{\mathrm{T}}$ categories, as well as in the DDBT passing and failing components of the $t \bar{t}$-enriched control region. The fit is performed separately for the three year periods. A combined fit over the three periods is performed for the final result. The theoretical uncertainties are correlated between different years. The test 
statistic chosen to determine the signal yield is based on the profile likelihood ratio [97]. Systematic uncertainties are incorporated into the analysis via nuisance parameters and treated according to the frequentist paradigm. The best-fit value of each signal strength parameter and an approximate $68 \%$ confidence level (CL) interval are extracted following the procedure described in section 3.2 of ref. [98].

Figure 3 shows the $m_{\mathrm{SD}}$ distributions in the combined data set for the DDBT passing and failing regions with the fitted background. The bottom panels of figure 3 show the difference between the data and the prediction from the background, divided by the statistical uncertainty in the data. These highlight the contributions from Higgs and V boson production in the failing and passing regions. The $\mathrm{W}$ boson contribution in the passing region is due to the misidentification of $\mathrm{W}(\mathrm{q} \overline{\mathrm{q}})$ decays by the DDBT. The agreement between the data and the signal-plus-background model is quantified with a Kolmogorov-Smirnov goodness-of-fit test [99], which yields a p-value of $17 \%$. In figure 4 , the $m_{\mathrm{SD}}$ distributions are shown for each $p_{\mathrm{T}}$ category in the passing region. The nuisance parameters related to the jet mass scale uncertainties, whose values extend up to $2 \mathrm{GeV}$ in the case of the $\mathrm{Z}$ boson as discussed in section 6 , do not significantly deviate from their pre-fit expectations.

To validate the substructure and $\mathrm{b}$ tagging techniques employed in this search, a maximum likelihood fit is performed using a model where the $\mathrm{Z}(\mathrm{q} \overline{\mathrm{q}})$ signal strength $\left(\mu_{\mathrm{Z}}\right)$ and $\mu_{\mathrm{H}}$ are left unconstrained. In the DDBT passing region, decays of the $\mathrm{Z}$ boson to $\mathrm{b} \overline{\mathrm{b}}$ constitute $79 \%$ of all $\mathrm{Z}$ decays. The product of cross section and branching fraction for the $\mathrm{Z}(\mathrm{q} \overline{\mathrm{q}})$ sample with $p_{\mathrm{T}}$ of the $\mathrm{Z}$ boson greater than $300 \mathrm{GeV}$ is $15.9 \mathrm{pb}$ and the product of acceptance and efficiency for events in which the $\mathrm{Z}$ boson is matched to the $\mathrm{H}(\mathrm{b} \overline{\mathrm{b}})$ candidate jet in the DDBT passing region is $0.41 \%$. The measured $\mu_{\mathrm{Z}}$ value is $1.01 \pm 0.05$ (stat) ${ }_{-0.15}^{+0.20}$ (syst) ${ }_{-0.09}^{+0.13}$ (theo). This demonstrates that the $\mathrm{Z}$ boson is clearly separable from the background. In this measurement, the dominant source of systematic uncertainty is the DDBT scale factor. For the remainder of results, $\mu_{\mathrm{Z}}$ is fixed to its expectation, with the corresponding uncertainties, as described in section 6 . Thus, the $\mathrm{Z}$ boson resonance is used to further constrain the DDBT scale factor in the Higgs boson measurements.

To extract the Higgs boson signal, three maximum likelihood fits are performed to the data, each with a different degree of reliance on the modeling of the Higgs boson $p_{\mathrm{T}}$ spectrum: the nominal inclusive fit using one $\mu_{\mathrm{H}}$ parameter for all $\mathrm{H}$ production modes and all jet $p_{\mathrm{T}}$ categories, an alternative fit using an independent $\mu_{\mathrm{H}}$ parameter for each $p_{\mathrm{T}}$ category for all $\mathrm{H}$ production modes to assess the compatibility among the $p_{\mathrm{T}}$ categories, and a fit which unfolds detector effects to present results for the $\mathrm{ggH}$ production mode at the generator level.

The product of cross section and branching fraction for all $\mathrm{H}(\mathrm{b} \overline{\mathrm{b}})$ processes with Higgs boson $p_{\mathrm{T}}>300 \mathrm{GeV}$ is $0.12 \mathrm{pb}$ and the product of acceptance and efficiency for events in which the $\mathrm{H}$ boson is matched to the $\mathrm{H}(\mathrm{b} \overline{\mathrm{b}})$ candidate jet in the DDBT passing region is $1.7 \%$. In the inclusive fit using the HJ-MiNLO sample as the $\mathrm{ggH}$ signal model and including the contributions from the other production modes, the measured $\mu_{\mathrm{H}}$ value is $3.7 \pm 1.2$ (stat $)_{-0.7}^{+0.8}$ (syst $)_{-0.5}^{+0.8}$ (theo). Upper limits at $95 \% \mathrm{CL}$ using the $\mathrm{CL}_{\mathrm{s}}$ criterion $[100,101]$ are obtained using asymptotic formulae [102]. The corresponding observed 

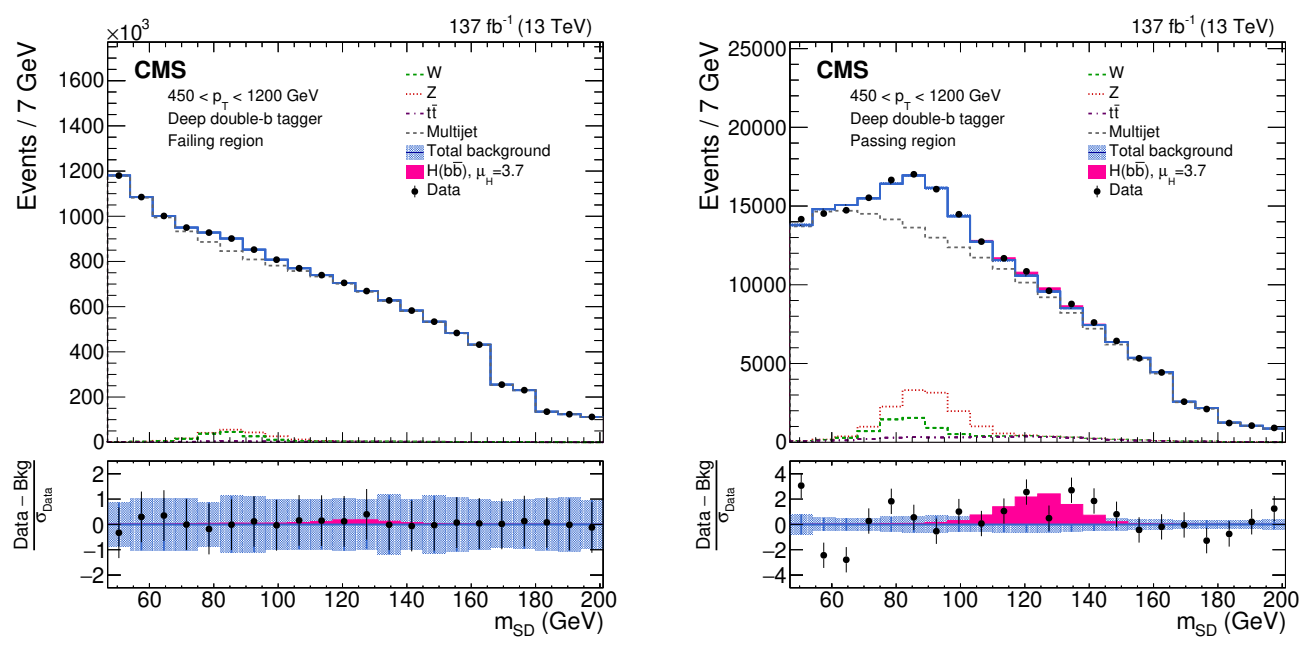

Figure 3. The observed and fitted background $m_{\mathrm{SD}}$ distributions for the DDBT failing (left) and passing (right) regions, combining all the $p_{\mathrm{T}}$ categories, and three data collection years. The fit is performed under the signal-plus-background hypothesis with one inclusive $\mathrm{H}(\mathrm{b} \overline{\mathrm{b}})$ signal strength parameter floating in all the $p_{\mathrm{T}}$ categories. Because of the finite $\rho$ acceptance, some $m_{\mathrm{SD}}$ bins within a given $p_{\mathrm{T}}$ category may be removed, giving rise to the steps at 166 and $180 \mathrm{GeV}$. The shaded blue band shows the systematic uncertainty in the total background prediction. The bottom panel shows the difference between the data and the total background prediction, divided by the statistical uncertainty in the data. In the failing region, the background model includes a free parameter for each $m_{\mathrm{SD}}$ bin, ensuring the nearly perfect agreement between the model and the data - this agreement is imperfect because the passing region is fit simultaneously and the global best fit is a balance between the two regions. Thus, the statistical uncertainty in the data gives rise to the systematic uncertainty in the background prediction. This is reflected in the fact that the error bar for the data and the uncertainty band for the background are approximately equal in size.

and expected upper limits on $\mu_{\mathrm{H}}$ at a $95 \%$ CL are 6.4 and 2.9 , respectively, while the observed and expected significances [103] with respect to the background-only hypothesis are $2.5 \sigma$ and $0.7 \sigma$. The measurement exhibits an excess over the SM expectation $\left(\mu_{\mathrm{H}}=1\right)$, with a significance of $1.9 \sigma$. Table 3 summarizes the measured signal strengths and significances for the Higgs and $\mathrm{Z}$ boson processes. The primary results using the $\mathrm{ggH} p_{\mathrm{T}}$ spectrum from HJ-MiNLO $[32,33]$ are shown, alongside results using the ggH $p_{\mathrm{T}}$ spectrum from ref. [23] for ease of comparison. The prediction used for the $\mathrm{ggH} p_{\mathrm{T}}$ spectrum in ref. [23] is different from that of HJ-MINLO in both shape and total cross section, which is primarily due to the different accuracy of finite top quark mass correction included in the simulation. In particular, the number of $\mathrm{ggH}$ signal events predicted by HJ-MiNLO in the fiducial region of the analysis is approximately a factor of two smaller than that of ref. [23], which is reflected in the fitted $\mu_{\mathrm{H}}$ values and their uncertainties. The fitted signal strength value and its uncertainty are sensitive to the $\mathrm{ggH}$ theoretical prediction and associated uncertainty, which are challenging to obtain in the high- $p_{\mathrm{T}}$ regime.

To assess the compatibility between the observed signal strengths in the different jet $p_{\mathrm{T}}$ categories, an alternative fit to the data is performed. In this fit, an independent $\mu_{\mathrm{H}}$ is assigned to each of the six reconstructed jet $p_{\mathrm{T}}$ bins. These signal strengths are 

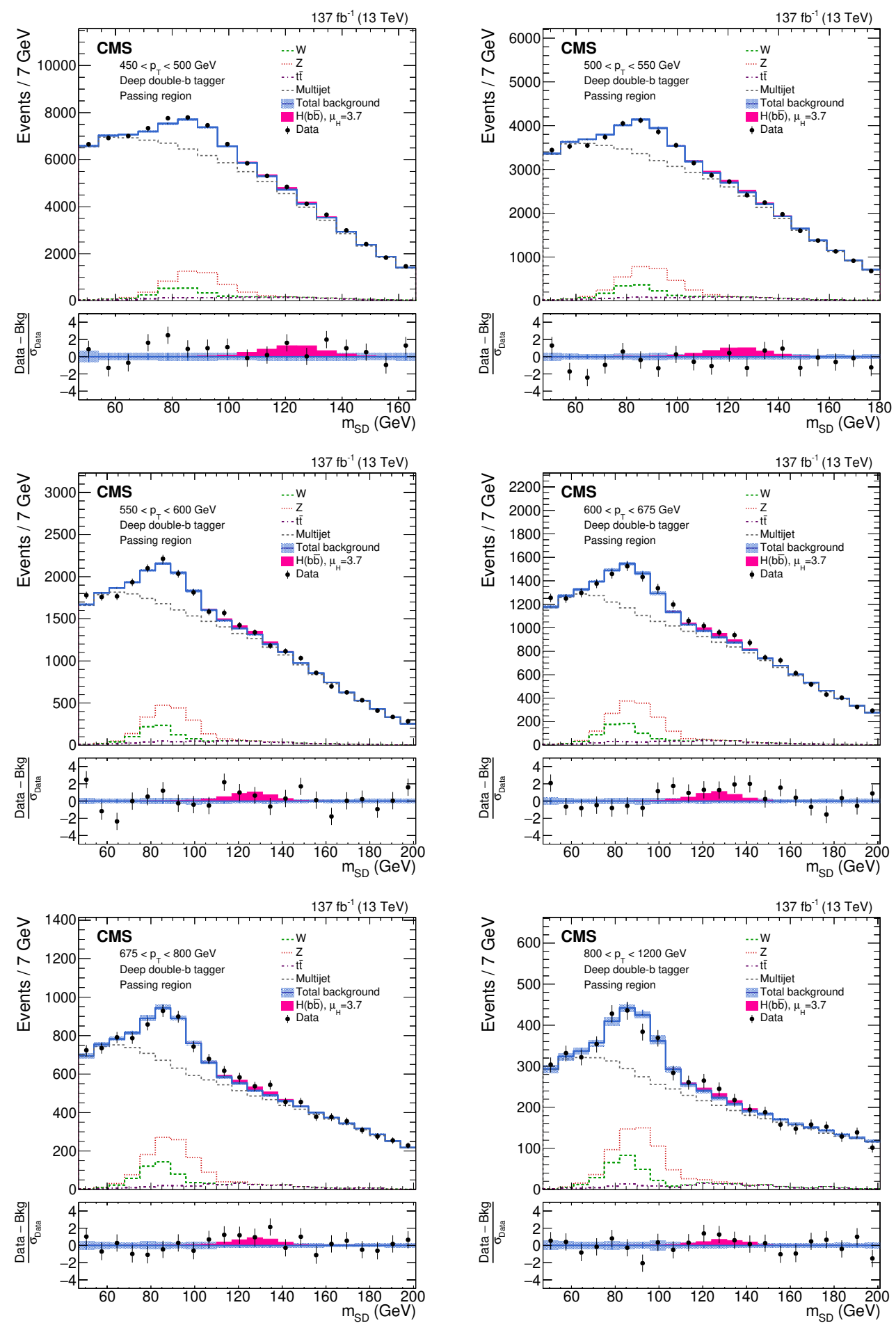

Figure 4. The observed and fitted background $m_{\mathrm{SD}}$ distributions in each $p_{\mathrm{T}}$ category in the DDBT passing regions. The fit is performed under the signal-plus-background hypothesis with one inclusive $\mathrm{H}(\mathrm{b} \overline{\mathrm{b}})$ signal strength parameter floating in all the $p_{\mathrm{T}}$ categories. The shaded blue band shows the systematic uncertainty in the total background prediction. The bottom panel shows the difference between the data and the total background prediction, divided by the statistical uncertainty in the data. 


\begin{tabular}{|lllll|}
\hline & 2016 & 2017 & 2018 & Combined \\
\hline Expected $\mu_{\mathrm{Z}}$ & $1.00_{-0.28}^{+0.38}$ & $1.00_{-0.29}^{+0.42}$ & $1.00_{-0.29}^{+0.43}$ & $1.00_{-0.19}^{+0.23}$ \\
Observed $\mu_{\mathrm{Z}}$ & $0.86_{-0.24}^{+0.32}$ & $1.11_{-0.33}^{+0.48}$ & $0.91_{-0.26}^{+0.37}$ & $1.01_{-0.20}^{+0.24}$ \\
HJ-MiNLO [32, 33] & & & & \\
Expected $\mu_{\mathrm{H}}$ & $1.0_{-3.5}^{+3.3}$ & $1.0 \pm 2.5$ & $1.0_{-2.4}^{+2.3}$ & $1.0 \pm 1.4$ \\
Observed $\mu_{\mathrm{H}}$ & $7.9_{-3.2}^{+3.4}$ & $4.8_{-2.5}^{+2.6}$ & $1.7 \pm 2.3$ & $3.7_{-1.5}^{+1.6}$ \\
Expected H significance $\left(\mu_{\mathrm{H}}=1\right)$ & $0.3 \sigma$ & $0.4 \sigma$ & $0.4 \sigma$ & $0.7 \sigma$ \\
Observed H significance & $2.4 \sigma$ & $1.9 \sigma$ & $0.7 \sigma$ & $2.5 \sigma$ \\
Expected UL $\mu_{\mathrm{H}}\left(\mu_{\mathrm{H}}=0\right)$ & $<6.8$ & $<5.0$ & $<4.7$ & $<2.9$ \\
Observed UL $\mu_{\mathrm{H}}$ & $<13.9$ & $<9.3$ & $<5.9$ & $<6.4$ \\
Ref. [23] H $p_{\mathrm{T}}$ spectrum & & & & \\
Expected $\mu_{\mathrm{H}}$ & $1.0 \pm 1.5$ & $1.0_{-1.0}^{+1.1}$ & $1.0_{-1.0}^{+1.1}$ & $1.0_{-0.6}^{+0.7}$ \\
Observed $\mu_{\mathrm{H}}$ & $4.0_{-1.6}^{+1.9}$ & $2.2_{-1.2}^{+1.4}$ & $1.1 \pm 1.1$ & $1.9_{-0.7}^{+0.9}$ \\
Expected H significance $\left(\mu_{\mathrm{H}}=1\right)$ & $0.7 \sigma$ & $0.9 \sigma$ & $1.0 \sigma$ & $1.7 \sigma$ \\
Observed H significance & $2.6 \sigma$ & $1.8 \sigma$ & $1.1 \sigma$ & $2.9 \sigma$ \\
Expected UL $\mu_{\mathrm{H}}\left(\mu_{\mathrm{H}}=0\right)$ & $<3.4$ & $<2.4$ & $<2.3$ & $<1.4$ \\
Observed UL $\mu_{\mathrm{H}}$ & $<7.4$ & $<4.6$ & $<3.2$ & $<3.4$ \\
\hline
\end{tabular}

Table 3. Fitted signal strength, and expected and observed significance of the Higgs and Z boson signals. The Higgs boson results are presented with two ggH signal models, one using the nominal HJ-MiNLO sample and the other simulated with the same procedure described in ref. [23]. The 95\% confidence level upper limit (UL) on the Higgs boson signal strength is also listed. In the results for the Higgs boson, the $\mathrm{Z}$ boson yield is fixed to the SM prediction value with the corresponding theoretical uncertainties to better constrain the data-to-simulation scale factor for the DDBT. For the expected and observed signal strengths of the $\mathrm{Z}$ boson, the Higgs boson signal strength is freely floating.

unconstrained in the fit and are varied simultaneously. All other parameters are profiled, as in the original fit. Figure 5 (left) illustrates the compatibility in the best fit signal strengths between the different $p_{\mathrm{T}}$ categories, showing an excess with respect to the SM expectation for categories with jet $p_{\mathrm{T}}$ above $550 \mathrm{GeV}$. Separately, the same exercise is performed with an independent $\mu_{\mathrm{Z}}$ in each $p_{\mathrm{T}}$ category. The fitted signal strengths, shown in figure 5 (right), are consistent with the SM expectation.

To facilitate comparisons with theoretical predictions, we isolate and remove the effects of limited detector acceptance and response to the $\mathrm{ggH}$ production cross section using a maximum-likelihood unfolding technique as described in section 5 of ref. [24]. In our treatment, the remaining Higgs boson production modes are assumed to occur at SM rates. The ggH signal is split into several bins according to the generated Higgs boson $p_{\mathrm{T}}\left(p_{\mathrm{T}}^{\mathrm{H}}\right)$, and each $p_{\mathrm{T}}^{\mathrm{H}}$ bin is considered as a separate process with a freely floating signal strength parameter in the likelihood model. The respective $p_{\mathrm{T}}^{\mathrm{H}}$ bins are 300-450, 450-650, and $>650 \mathrm{GeV}$. This binning choice follows the simplified template cross section (STXS) recommendation [104]. As the minimum reconstructed jet $p_{\mathrm{T}}$ is $450 \mathrm{GeV}$, a negligible signal 

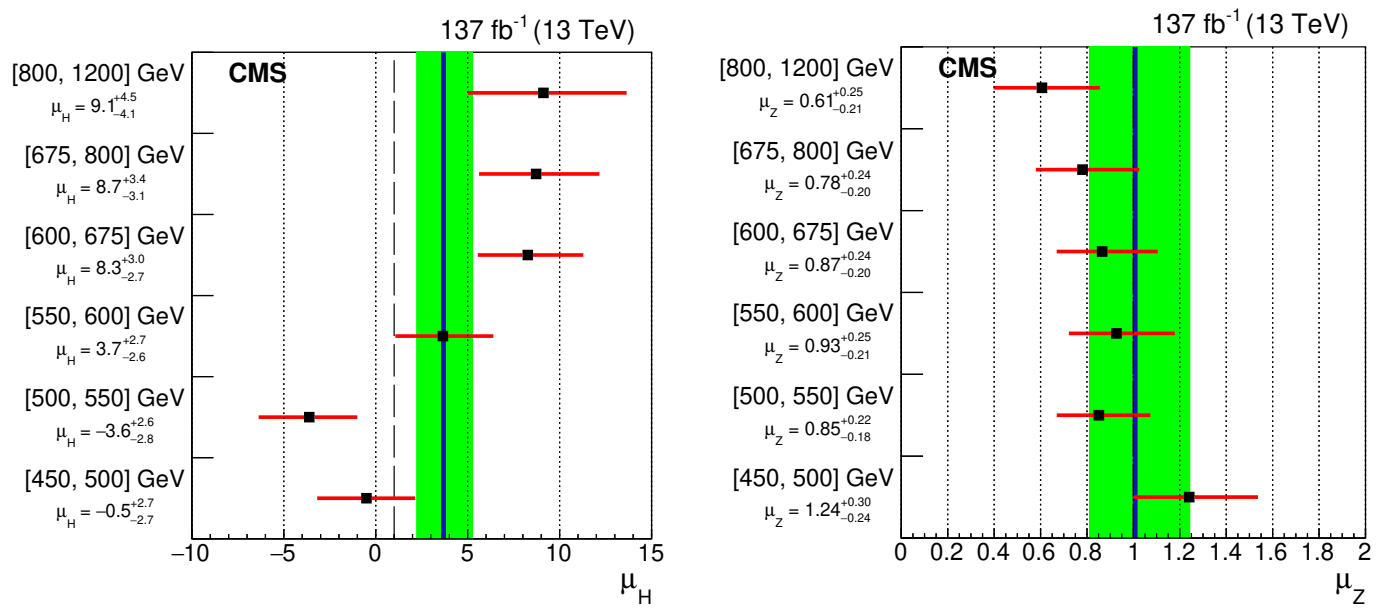

Figure 5. The best-fit signal strength $\mu_{\mathrm{H}}$ (black squares) and uncertainty (red lines) per $p_{\mathrm{T}}$ category based on the HJ-MiNLO [32, 33] prediction (left) and the same for $\mu_{\text {Z }}$ (right). The dashed black line indicates the SM expectation. The solid blue line and green band represents the combined best-fit signal strength and uncertainty, respectively, of $\mu_{\mathrm{H}}=3.7_{-1.5}^{+1.6}$ or $\mu_{\mathrm{Z}}=1.01_{-0.20}^{+0.24}$ extracted from a simultaneous fit of all categories.

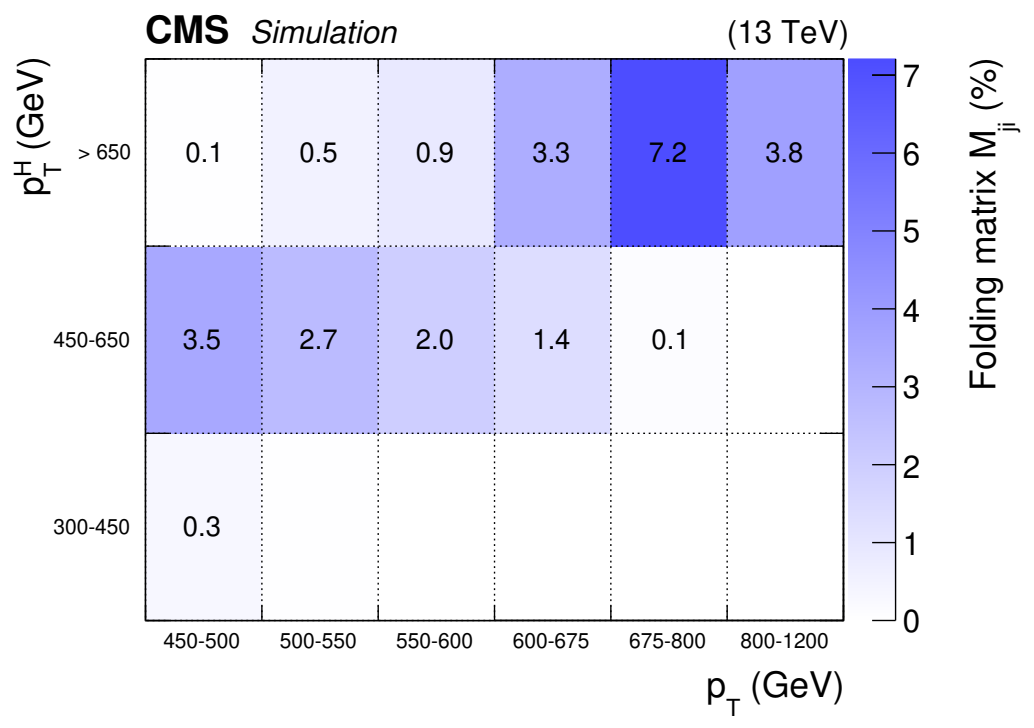

Figure 6. The folding matrix $M_{j i}$, defined as the product of the acceptance and the efficiency as a percentage for an $\mathrm{H}(\mathrm{b} \overline{\mathrm{b}})$ event in $p_{\mathrm{T}}^{\mathrm{H}}$ bin $j$ to be found in jet $p_{\mathrm{T}}$ bin $i$, for the ggH HJ-MiNLO simulation.

contribution is expected from events with $p_{\mathrm{T}}^{\mathrm{H}}<300 \mathrm{GeV}$. The folding matrix $M_{j i}$, defined as the product of the acceptance and the efficiency for an $\mathrm{H}(\mathrm{b} \overline{\mathrm{b}})$ event in $p_{\mathrm{T}}^{\mathrm{H}}$ bin $j$ to be found in jet $p_{\mathrm{T}}$ bin $i$, is shown in figure 6 for the ggH HJ-MINLO simulation. This matrix is found to be well-conditioned. Therefore, we omit any regularization in the unfolding procedure [105].

The ggH fiducial cross section in each STXS $p_{\mathrm{T}}^{\mathrm{H}}$ bin is then extracted by scaling the cross section found in simulation, imposing no selection requirements other than those 


\begin{tabular}{|c|c|c|c|c|c|c|}
\hline$p_{\mathrm{T}}^{\mathrm{H}}(\mathrm{GeV})$ & \multicolumn{2}{|r|}{$300-450$} & \multicolumn{2}{|r|}{$450-650$} & \multicolumn{2}{|r|}{$>650$} \\
\hline Measured & 580 & \pm 790 & 5 & \pm 43 & 29 & \pm 11 \\
\hline Niteasured & & \pm 720 (stat) \pm 350 (syst) & & \pm 37 (stat) \pm 22 (syst) & & \pm 9 (stat) \pm 7 (syst) \\
\hline LHCHXSWG [33] & & - & 16.0 & $\begin{array}{l}+1.7 \\
-2.0\end{array}$ & 2.1 & ${ }_{-0.3}^{+0.2}$ \\
\hline HJ-MINLO [32] & 89 & $\begin{array}{l}+20 \\
-18\end{array}$ & 13.5 & $\begin{array}{l}+3.0 \\
-2.7\end{array}$ & 1.9 & \pm 0.4 \\
\hline Ref. [23] & 152 & \pm 46 & 34 & \pm 10 & 7.6 & \pm 3.0 \\
\hline
\end{tabular}

Table 4. Measured and predicted ggH differential fiducial cross section as a function of Higgs boson $p_{\mathrm{T}}$. All cross sections are in units of fb. The cumulative cross section predictions from ref. [33] are converted to differential cross section predictions by subtraction assuming the cumulative cross section uncertainties are fully correlated.

\begin{tabular}{|lccc|}
\hline$p_{\mathrm{T}}^{\mathrm{H}}(\mathrm{GeV})$ & $300-450$ & $450-650$ & $>650$ \\
\hline $300-450$ & 1.0 & -0.18 & -0.002 \\
$450-650$ & -0.18 & 1.0 & 0.06 \\
$>650$ & -0.002 & 0.06 & 1.0 \\
\hline
\end{tabular}

Table 5. Correlation coefficients between the three $p_{\mathrm{T}}^{\mathrm{H}}$ bins of the unfolded gg $\mathrm{H}$ differential cross section measurement.

on $p_{\mathrm{T}}^{\mathrm{H}}$, by the corresponding signal strength parameter. The uncertainty in this value is taken from the correspondingly scaled signal strength uncertainty. For the theoretical uncertainties, only those that affect the acceptance of signal events into the reconstructed selection are taken into account. Based on the envelope of acceptance values from varying the renormalization and factorization scales by factors of two, this theoretical acceptance uncertainty is estimated to be $2 \%$. We verify that this unfolding procedure is unbiased through signal injection studies.

The result of this unfolding procedure is shown in figure 7 and table 4, along with the predicted cross sections from ref. [33] and the predictions of the signal event generators described in section 3. The correlation coefficients among the three $p_{\mathrm{T}}^{\mathrm{H}}$ bins are shown in table 5. The measured cross section uncertainty in the first $p_{\mathrm{T}}^{\mathrm{H}}$ bin is larger because of limited acceptance. The first and second $p_{\mathrm{T}}^{\mathrm{H}}$ bins have a mild anti-correlation, primarily because of the imperfect jet energy response of the detector, which inflates the corresponding per-bin uncertainties in the unfolded cross section. The observed cross section in the third $p_{\mathrm{T}}^{\mathrm{H}}$ bin has a smaller relative uncertainty than that in the second bin because of the larger magnitude of the central value in that bin. With respect to the SM, the upward deviation of the cross section in the third $p_{\mathrm{T}}^{\mathrm{H}}$ bin, when profiling the other two, corresponds to a local significance of $2.6 \sigma$. When considering all three cross section parameters of interest simultaneously, the total deviation from the SM corresponds to a significance of $1.9 \sigma$.

\section{Summary}

An inclusive search for the standard model (SM) Higgs boson decaying to a bottom quarkantiquark pair and reconstructed as a single large-radius jet with transverse momentum $p_{\mathrm{T}}>450 \mathrm{GeV}$ has been presented. The search uses a data sample of proton-proton collisions at $\sqrt{s}=13 \mathrm{TeV}$, corresponding to an integrated luminosity of $137 \mathrm{fb}^{-1}$. The associated 


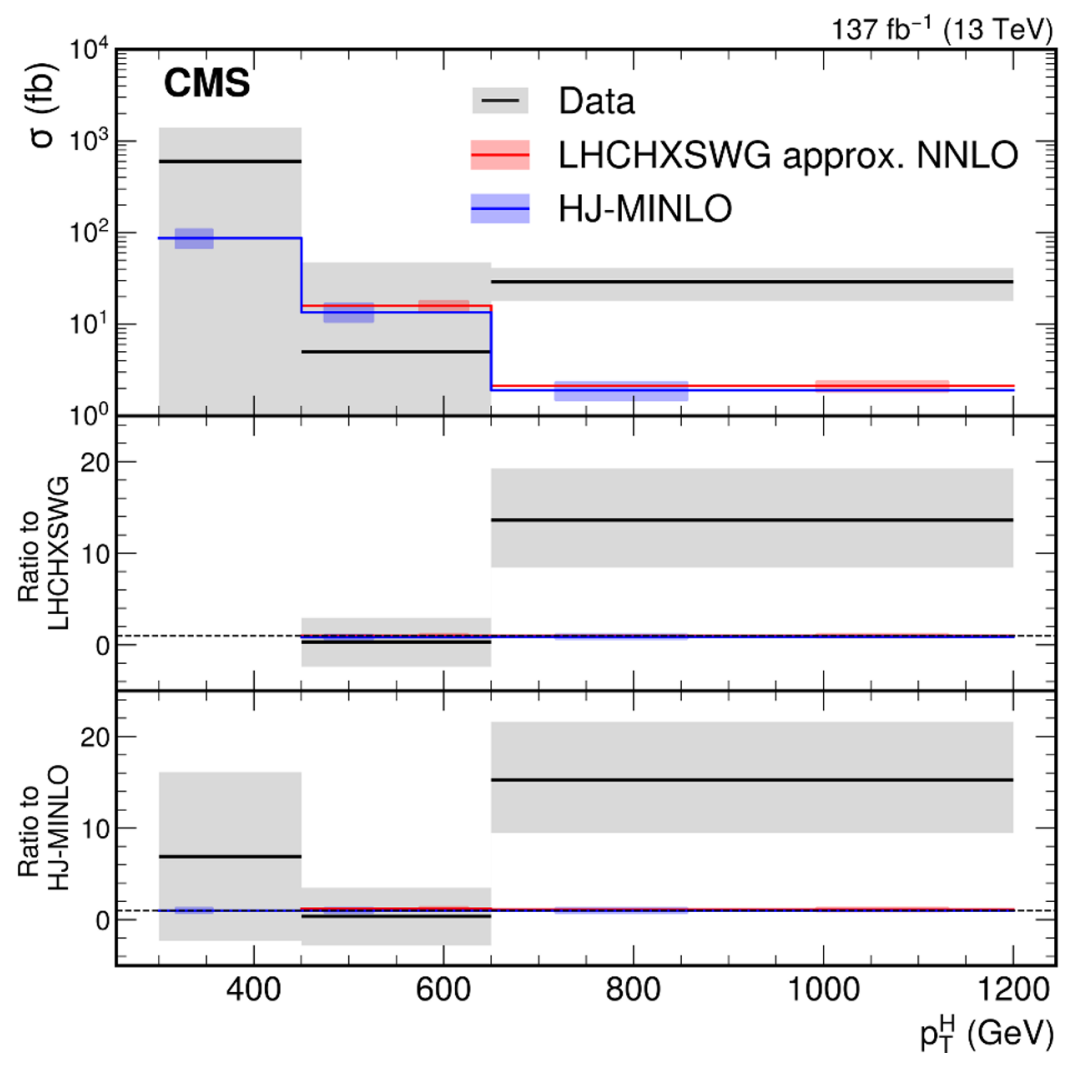

Figure 7. Measured ggH differential fiducial cross section as a function of Higgs boson $p_{\mathrm{T}}$ shown in black, in comparison to the predictions of ref. [33], shown in red, and HJ-MiNLO [32], shown in blue. The two predictions are nearly identical. The larger gray band shows the total uncertainty in the measured cross section while the red and blue hatched bands show the uncertainties in the predictions of ref. [33] and HJ-MiNLO, respectively. In the bottom two panels, the dotted line corresponds to a ratio of one. The relative uncertainties in the predictions of ref. [33] and HJ-MiNLO are approximately 10 and 20\%, respectively.

production of a $\mathrm{Z}$ boson and jets is used to validate the method and is measured to be consistent with the SM prediction. The inclusive Higgs boson signal strength is measured to be $\mu_{\mathrm{H}}=3.7 \pm 1.2(\text { stat })_{-0.7}^{+0.8}$ (syst) $)_{-0.5}^{+0.8}$ (theo) $=3.7_{-1.5}^{+1.6}$, based on the theoretical prediction from the HJ-MiNLO generator for the gluon fusion production mode. The measured $\mu_{\mathrm{H}}$ corresponds to an observed significance of 2.5 standard deviations $(\sigma)$ with respect to the background-only hypothesis, while the expected significance of the SM signal is $0.7 \sigma$. The significance of the observed excess with respect to the SM expectation is $1.9 \sigma$. With respect to the previous CMS result, the relative precision of the $\mu_{\mathrm{H}}$ measurement improves by approximately a factor of two because of the increased integrated luminosity, an improved $\mathrm{b}$ tagging technique based on a deep neural network, and smaller theoretical uncertainties. Finally, the differential cross section for the $p_{\mathrm{T}}$ of a Higgs boson produced through gluon fusion, assuming the other production modes occur at the SM rates, in the phase space regions recommended by the LHC simplified template cross section framework has also been presented. An excess is seen for Higgs boson $p_{\mathrm{T}}>650 \mathrm{GeV}$ with a local significance of $2.6 \sigma$ with respect to the SM expectation including the Higgs boson. 


\section{Acknowledgments}

We congratulate our colleagues in the CERN accelerator departments for the excellent performance of the LHC and thank the technical and administrative staffs at CERN and at other CMS institutes for their contributions to the success of the CMS effort. In addition, we gratefully acknowledge the computing centers and personnel of the Worldwide LHC Computing Grid for delivering so effectively the computing infrastructure essential to our analyses. Finally, we acknowledge the enduring support for the construction and operation of the LHC and the CMS detector provided by the following funding agencies: BMBWF and FWF (Austria); FNRS and FWO (Belgium); CNPq, CAPES, FAPERJ, FAPERGS, and FAPESP (Brazil); MES (Bulgaria); CERN; CAS, MoST, and NSFC (China); COLCIENCIAS (Colombia); MSES and CSF (Croatia); RIF (Cyprus); SENESCYT (Ecuador); MoER, ERC IUT, PUT and ERDF (Estonia); Academy of Finland, MEC, and HIP (Finland); CEA and CNRS/IN2P3 (France); BMBF, DFG, and HGF (Germany); GSRT (Greece); NKFIA (Hungary); DAE and DST (India); IPM (Iran); SFI (Ireland); INFN (Italy); MSIP and NRF (Republic of Korea); MES (Latvia); LAS (Lithuania); MOE and UM (Malaysia); BUAP, CINVESTAV, CONACYT, LNS, SEP, and UASLP-FAI (Mexico); MOS (Montenegro); MBIE (New Zealand); PAEC (Pakistan); MSHE and NSC (Poland); FCT (Portugal); JINR (Dubna); MON, RosAtom, RAS, RFBR, and NRC KI (Russia); MESTD (Serbia); SEIDI, CPAN, PCTI, and FEDER (Spain); MOSTR (Sri Lanka); Swiss Funding Agencies (Switzerland); MST (Taipei); ThEPCenter, IPST, STAR, and NSTDA (Thailand); TUBITAK and TAEK (Turkey); NASU (Ukraine); STFC (United Kingdom); DOE and NSF (U.S.A.).

Individuals have received support from the Marie-Curie program and the European Research Council and Horizon 2020 Grant, contract Nos. 675440, 752730, and 765710 (European Union); the Leventis Foundation; the A.P. Sloan Foundation; the Alexander von Humboldt Foundation; the Belgian Federal Science Policy Office; the Fonds pour la Formation à la Recherche dans l'Industrie et dans l'Agriculture (FRIA-Belgium); the Agentschap voor Innovatie door Wetenschap en Technologie (IWT-Belgium); the F.R.S.-FNRS and FWO (Belgium) under the "Excellence of Science — EOS" — be.h project n. 30820817; the Beijing Municipal Science \& Technology Commission, No. Z191100007219010; the Ministry of Education, Youth and Sports (MEYS) of the Czech Republic; the Deutsche Forschungsgemeinschaft (DFG) under Germany's Excellence Strategy — EXC 2121 "Quantum Universe" - 390833306; the Lendület ("Momentum") Program and the János Bolyai Research Scholarship of the Hungarian Academy of Sciences, the New National Excellence Program ÚNKP, the NKFIA research grants 123842, 123959, 124845, 124850, 125105, 128713, 128786, and 129058 (Hungary); the Council of Science and Industrial Research, India; the HOMING PLUS program of the Foundation for Polish Science, cofinanced from European Union, Regional Development Fund, the Mobility Plus program of the Ministry of Science and Higher Education, the National Science Center (Poland), contracts Harmonia 2014/14/M/ST2/00428, Opus 2014/13/B/ST2/02543, 2014/15/B/ST2/03998, and 2015/19/B/ST2/02861, Sonata-bis 2012/07/E/ST2/01406; the National Priorities Research Program by Qatar National Research Fund; the Ministry of Science and Higher 
Education, project no. 02.a03.21.0005 (Russia); the Tomsk Polytechnic University Competitiveness Enhancement Program and "Nauka" Project FSWW-2020-0008 (Russia); the Programa Estatal de Fomento de la Investigación Científica y Técnica de Excelencia María de Maeztu, grant MDM-2015-0509 and the Programa Severo Ochoa del Principado de Asturias; the Thalis and Aristeia programs cofinanced by EU-ESF and the Greek NSRF; the Rachadapisek Sompot Fund for Postdoctoral Fellowship, Chulalongkorn University and the Chulalongkorn Academic into Its 2nd Century Project Advancement Project (Thailand); the Kavli Foundation; the Nvidia Corporation; the SuperMicro Corporation; the Welch Foundation, contract C-1845; and the Weston Havens Foundation (U.S.A.).

Open Access. This article is distributed under the terms of the Creative Commons Attribution License (CC-BY 4.0), which permits any use, distribution and reproduction in any medium, provided the original author(s) and source are credited.

\section{References}

[1] ATLAS collaboration, Observation of a new particle in the search for the Standard Model Higgs boson with the ATLAS detector at the LHC, Phys. Lett. B 716 (2012) 1 [arXiv: 1207.7214] [INSPIRE].

[2] CMS collaboration, Observation of a new boson at a mass of $125 \mathrm{GeV}$ with the CMS experiment at the LHC, Phys. Lett. B $\mathbf{7 1 6}$ (2012) 30 [arXiv:1207.7235] [INSPIRE].

[3] CMS collaboration, Observation of a new boson with mass near $125 \mathrm{GeV}$ in pp collisions at $\sqrt{s}=7$ and $8 \mathrm{TeV}$, JHEP 06 (2013) 081 [arXiv:1303.4571] [INSPIRE].

[4] A. Salam, Weak and electromagnetic interactions, in Elementary particle physics: relativistic groups and analyticity, N. Svartholm ed., Almqvist \& Wiksell, Stockholm, Sweden (1968).

[5] S.L. Glashow, Partial symmetries of weak interactions, Nucl. Phys. 22 (1961) 579 [INSPIRE].

[6] S. Weinberg, A model of leptons, Phys. Rev. Lett. 19 (1967) 1264 [INSPIRE].

[7] F. Englert and R. Brout, Broken symmetry and the mass of gauge vector mesons, Phys. Rev. Lett. 13 (1964) 321 [INSPIRE].

[8] W.F. Baker et al., Large angle proton proton scattering at 30 BeV, Phys. Rev. Lett. 12 (1964) 132 [INSPIRE].

[9] P.W. Higgs, Broken symmetries and the masses of gauge bosons, Phys. Rev. Lett. 13 (1964) 508 [INSPIRE].

[10] P.W. Higgs, Spontaneous symmetry breakdown without massless bosons, Phys. Rev. Lett. 145 (1966) 1156 [INSPIRE].

[11] G.S. Guralnik, C.R. Hagen and T.W.B. Kibble, Global conservation laws and massless particles, Phys. Rev. Lett. 13 (1964) 585 [INSPIRE].

[12] CMS collaboration, Observation of Higgs boson decay to bottom quarks, Phys. Rev. Lett. 121 (2018) 121801 [arXiv: 1808.08242] [INSPIRE].

[13] ATLAS collaboration, Observation of $H \rightarrow b \bar{b}$ decays and $V H$ production with the ATLAS detector, Phys. Lett. B 786 (2018) 59 [arXiv:1808.08238] [INSPIRE]. 
[14] LHC Higgs Cross Section Working Group collaboration, Handbook of LHC Higgs Cross Sections: 4. Deciphering the nature of the Higgs sector, CYRM-2017-002 [arXiv: 1610.07922] [INSPIRE].

[15] M.H. Seymour, Tagging a heavy Higgs boson, in the proceedings of the ECFA Large Hadron Collider Workshop, October 4-9, Aachen, Germany (1990) [INSPIRE].

[16] M.H. Seymour, Searches for new particles using cone and cluster jet algorithms: a comparative study, Z. Phys. C 62 (1994) 127 [InSPIRE].

[17] M.H. Seymour, The average number of subjets in a hadron collider jet, Nucl. Phys. B 421 (1994) 545 [INSPIRE].

[18] J.M. Butterworth, B.E. Cox and J.R. Forshaw, $W W$ scattering at the CERN LHC, Phys. Rev. D 65 (2002) 096014 [hep-ph/0201098] [INSPIRE].

[19] J.M. Butterworth, A.R. Davison, M. Rubin and G.P. Salam, Jet substructure as a new Higgs search channel at the LHC, Phys. Rev. Lett. 100 (2008) 242001 [arXiv:0802.2470] [INSPIRE].

[20] CMS collaboration, Identification of heavy-flavour jets with the CMS detector in pp collisions at $13 \mathrm{TeV}, 2018$ JINST 13 P05011 [arXiv:1712.07158] [INSPIRE].

[21] CMS collaboration, Performance of deep tagging algorithms for boosted double quark jet topology in proton-proton collisions at $13 \mathrm{TeV}$ with the phase- $0 \mathrm{CMS}$ detector, CMS-DP-2018-046.

[22] ATLAS collaboration, Identification of boosted Higgs bosons decaying into b-quark pairs with the ATLAS detector at 13 TeV, Eur. Phys. J. C 79 (2019) 836 [arXiv:1906.11005] [INSPIRE].

[23] CMS collaboration, Inclusive search for a highly boosted Higgs boson decaying to a bottom quark-antiquark pair, Phys. Rev. Lett. 120 (2018) 071802 [arXiv:1709.05543] [INSPIRE].

[24] CMS collaboration, Measurement and interpretation of differential cross sections for Higgs boson production at $\sqrt{s}=13 \mathrm{TeV}$, Phys. Lett. B 792 (2019) 369 [arXiv:1812.06504] [INSPIRE].

[25] C. Grojean, E. Salvioni, M. Schlaffer and A. Weiler, Very boosted Higgs in gluon fusion, JHEP 05 (2014) 022 [arXiv: 1312.3317] [InSPIRE].

[26] S. Dawson, I.M. Lewis and M. Zeng, Usefulness of effective field theory for boosted Higgs production, Phys. Rev. D 91 (2015) 074012 [arXiv:1501.04103] [INSPIRE].

[27] M. Schlaffer, M. Spannowsky, M. Takeuchi, A. Weiler and C. Wymant, Boosted Higgs shapes, Eur. Phys. J. C 74 (2014) 3120 [arXiv:1405.4295] [INSPIRE].

[28] M. Grazzini, A. Ilnicka, M. Spira and M. Wiesemann, Effective field theory for Higgs properties parametrisation: the transverse momentum spectrum case, in the proceedings of the $52^{\text {nd }}$ Rencontres de Moriond on QCD and High Energy Interactions, March 25-April 1, La Thuile, Italy (2017), arXiv:1705.05143 [INSPIRE].

[29] M. Grazzini, A. Ilnicka, M. Spira and M. Wiesemann, Modeling BSM effects on the Higgs transverse-momentum spectrum in an EFT approach, JHEP 03 (2017) 115 [arXiv: 1612.00283] [INSPIRE].

[30] F. Bishara, U. Haisch, P.F. Monni and E. Re, Constraining light-quark Yukawa couplings from Higgs distributions, Phys. Rev. Lett. 118 (2017) 121801 [arXiv:1606.09253] [INSPIRE]. 
[31] Y.-Y. Li, R. Nicolaidou and S. Paganis, Exclusion of heavy, broad resonances from precise measurements of $W Z$ and $V H$ final states at the LHC, Eur. Phys. J. C 79 (2019) 348 [arXiv: 1904.03995] [INSPIRE].

[32] K. Hamilton, P. Nason, C. Oleari and G. Zanderighi, Merging $H / W / Z+0$ and 1 jet at NLO with no merging scale: a path to parton shower + NNLO matching, JHEP 05 (2013) 082 [arXiv: 1212.4504] [INSPIRE].

[33] K. Becker et al., Precise predictions for boosted Higgs production, arXiv:2005.07762 [INSPIRE].

[34] T. Neumann, NLO Higgs +jet production at large transverse momenta including top quark mass effects, J. Phys. Comm. 2 (2018) 095017 [arXiv: 1802. 02981] [INSPIRE].

[35] S.P. Jones, M. Kerner and G. Luisoni, Next-to-Leading-Order QCD corrections to Higgs boson plus jet production with full top-quark mass dependence, Phys. Rev. Lett. 120 (2018) 162001 [arXiv: 1802.00349] [INSPIRE].

[36] J.M. Lindert, K. Kudashkin, K. Melnikov and C. Wever, Higgs bosons with large transverse momentum at the LHC, Phys. Lett. B 782 (2018) 210 [arXiv:1801.08226] [InSPIRE].

[37] CMS collaboration, The CMS trigger system, 2017 JINST 12 P01020 [arXiv:1609.02366] [INSPIRE].

[38] CMS collaboration, The CMS experiment at the CERN LHC, 2008 JINST 3 S08004 [INSPIRE].

[39] GEANT4 collaboration, GEANT4 - A simulation toolkit, Nucl. Instrum. Meth. A $\mathbf{5 0 6}$ (2003) 250 [INSPIRE].

[40] J. Alwall et al., The automated computation of tree-level and next-to-leading order differential cross sections, and their matching to parton shower simulations, JHEP $\mathbf{0 7}$ (2014) 079 [arXiv: 1405.0301] [INSPIRE].

[41] J. Alwall et al., Comparative study of various algorithms for the merging of parton showers and matrix elements in hadronic collisions, Eur. Phys. J. C 53 (2008) 473 [arXiv:0706.2569] [INSPIRE].

[42] P. Nason, A New method for combining NLO QCD with shower Monte Carlo algorithms, JHEP 11 (2004) 040 [hep-ph/0409146] [INSPIRE].

[43] S. Frixione, P. Nason and C. Oleari, Matching NLO QCD computations with Parton Shower simulations: the POWHEG method, JHEP 11 (2007) 070 [arXiv: 0709.2092] [INSPIRE].

[44] S. Alioli, P. Nason, C. Oleari and E. Re, A general framework for implementing NLO calculations in shower Monte Carlo programs: the POWHEG BOX, JHEP 06 (2010) 043 [arXiv: 1002.2581] [INSPIRE].

[45] S. Frixione, P. Nason and G. Ridolfi, A positive-weight next-to-leading-order Monte Carlo for heavy flavour hadroproduction, JHEP 09 (2007) 126 [arXiv:0707.3088] [INSPIRE].

[46] R. Frederix, E. Re and P. Torrielli, Single-top t-channel hadroproduction in the four-flavour scheme with POWHEG and aMC@NLO, JHEP 09 (2012) 130 [arXiv:1207.5391] [INSPIRE].

[47] E. Re, Single-top Wt-channel production matched with parton showers using the POWHEG method, Eur. Phys. J. C 71 (2011) 1547 [arXiv: 1009.2450] [INSPIRE].

[48] T. Sjöstrand et al., An introduction to PYTHIA 8.2, Comput. Phys. Commun. 191 (2015) 159 [arXiv:1410.3012] [INSPIRE]. 
[49] R. Frederix and S. Frixione, Merging meets matching in MC@NLO, JHEP 12 (2012) 061 [arXiv:1209.6215] [INSPIRE].

[50] S. Kallweit, J.M. Lindert, P. Maierhöfer, S. Pozzorini and M. Schönherr, NLO electroweak automation and precise predictions for W+multijet production at the LHC, JHEP 04 (2015) 012 [arXiv: 1412.5157] [INSPIRE].

[51] S. Kallweit, J.M. Lindert, P. Maierhofer, S. Pozzorini and M. Schönherr, $N L O Q C D+E W$ predictions for $V+$ jets including off-shell vector-boson decays and multijet merging, JHEP 04 (2016) 021 [arXiv: 1511.08692] [INSPIRE].

[52] S. Kallweit, J.M. Lindert, S. Pozzorini, M. Schönherr and P. Maierhöfer, $N L O Q C D+E W$ automation and precise predictions for $V+$ multijet production, in the proceedings of $50^{\text {th }}$ Rencontres de Moriond on QCD and high energy interactions, March 21-28, La Thuile, Italy (2015) [arXiv: 1505.05704] [INSPIRE].

[53] J.M. Lindert et al., Precise predictions for $V+$ jets dark matter backgrounds, Eur. Phys. J. $C 77$ (2017) 829 [arXiv: 1705.04664] [INSPIRE].

[54] J.M. Campbell and R.K. Ellis, MCFM for the Tevatron and the LHC, Nucl. Phys. B Proc. Suppl. 205-206 (2010) 10 [arXiv:1007.3492] [INSPIRE].

[55] G. Luisoni, P. Nason, C. Oleari and F. Tramontano, $H W^{ \pm} / H Z+O$ and 1 jet at NLO with the POWHEG BOX interfaced to GoSam and their merging within MiNLO, JHEP 10 (2013) 083 [arXiv: 1306.2542] [INSPIRE].

[56] E. Bagnaschi, G. Degrassi, P. Slavich and A. Vicini, Higgs production via gluon fusion in the POWHEG approach in the SM and in the MSSM, JHEP 02 (2012) 088 [arXiv:1111.2854] [INSPIRE].

[57] P. Nason and C. Oleari, NLO Higgs boson production via vector-boson fusion matched with shower in POWHEG, JHEP 02 (2010) 037 [arXiv:0911.5299] [INSPIRE].

[58] H.B. Hartanto, B. Jager, L. Reina and D. Wackeroth, Higgs boson production in association with top quarks in the POWHEG BOX, Phys. Rev. D 91 (2015) 094003 [arXiv: 1501.04498] [INSPIRE].

[59] M. Cacciari, F.A. Dreyer, A. Karlberg, G.P. Salam and G. Zanderighi, Fully differential vector-boson-fusion Higgs production at next-to-next-to-leading order, Phys. Rev. Lett. 115 (2015) 082002 [Erratum ibid. 120 (2018) 139901] [arXiv:1506.02660] [INSPIRE].

[60] F.A. Dreyer and A. Karlberg, Vector-boson fusion Higgs production at three loops in QCD, Phys. Rev. Lett. 117 (2016) 072001 [arXiv:1606.00840] [INSPIRE].

[61] CMS collaboration, Event generator tunes obtained from underlying event and multiparton scattering measurements, Eur. Phys. J. C 76 (2016) 155 [arXiv: 1512.00815] [INSPIRE].

[62] CMS collaboration, Extraction and validation of a new set of CMS PYTHIA8 tunes from underlying-event measurements, Eur. Phys. J. C 80 (2020) 4 [arXiv:1903.12179] [INSPIRE].

[63] CMS collaboration, Investigations of the impact of the parton shower tuning in PYTHIA 8 in the modelling of $t \bar{t}$ at $\sqrt{s}=8$ and $13 \mathrm{TeV}$, CMS-PAS-TOP-16-021 (2016) [INSPIRE].

[64] NNPDF collaboration, Parton distributions for the LHC Run II, JHEP 04 (2015) 040 [arXiv: 1410.8849] [INSPIRE].

[65] NNPDF collaboration, Parton distributions from high-precision collider data, Eur. Phys. J. C 77 (2017) 663 [arXiv:1706.00428] [InSPIRE]. 
[66] CMS collaboration, Particle-flow reconstruction and global event description with the CMS detector, 2017 JINST 12 P10003 [arXiv:1706.04965] [INSPIRE].

[67] M. Cacciari, G.P. Salam and G. Soyez, The anti- $k_{t}$ jet clustering algorithm, JHEP 04 (2008) 063 [arXiv: 0802.1189] [InSPIRE].

[68] M. Cacciari, G.P. Salam and G. Soyez, FastJet user manual, Eur. Phys. J. C 72 (2012) 1896 [arXiv:1111.6097] [INSPIRE].

[69] D. Bertolini, P. Harris, M. Low and N. Tran, Pileup per particle identification, JHEP 10 (2014) 059 [arXiv: 1407.6013] [INSPIRE].

[70] CMS collaboration, Pileup mitigation at CMS in $13 \mathrm{TeV}$ data, 2020 JINST 15 P09018 [arXiv: 2003. 00503] [INSPIRE].

[71] CMS collaboration, Jet energy scale and resolution in the CMS experiment in pp collisions at $8 \mathrm{TeV}, 2017$ JINST 12 P02014 [arXiv:1607.03663] [INSPIRE].

[72] CMS collaboration, Jet algorithms performance in $13 \mathrm{TeV}$ data, CMS-PAS-JME-16-003 (2017) [INSPIRE].

[73] D. Krohn, J. Thaler and L.-T. Wang, Jet trimming, JHEP 02 (2010) 084 [arXiv:0912.1342] [INSPIRE].

[74] CMS collaboration, Search for neutral Higgs bosons decaying to tau pairs in pp collisions at $\sqrt{s}=7$ TeV, Phys. Lett. B 713 (2012) 68 [arXiv:1202.4083] [INSPIRE].

[75] CMS collaboration, Performance of electron reconstruction and selection with the CMS detector in proton-proton collisions at $\sqrt{s}=8 \mathrm{TeV}, 2015$ JINST $10 \mathrm{P} 06005$ [arXiv: 1502. 02701] [INSPIRE].

[76] CMS collaboration, Performance of the CMS muon detector and muon reconstruction with proton-proton collisions at $\sqrt{s}=13 \mathrm{TeV}, 2018$ JINST 13 P06015 [arXiv:1804.04528] [INSPIRE].

[77] A.J. Larkoski, S. Marzani, G. Soyez and J. Thaler, Soft drop, JHEP 05 (2014) 146 [arXiv: 1402.2657] [INSPIRE].

[78] M. Dasgupta, A. Fregoso, S. Marzani and G.P. Salam, Towards an understanding of jet substructure, JHEP 09 (2013) 029 [arXiv:1307.0007] [INSPIRE].

[79] J. Dolen, P. Harris, S. Marzani, S. Rappoccio and N. Tran, Thinking outside the ROCs: Designing Decorrelated Taggers (DDT) for jet substructure, JHEP 05 (2016) 156 [arXiv: 1603.00027] [INSPIRE].

[80] I. Moult, L. Necib and J. Thaler, New angles on energy correlation functions, JHEP 12 (2016) 153 [arXiv: 1609. 07483] [inSPIRE].

[81] A.J. Larkoski, G.P. Salam and J. Thaler, Energy correlation functions for jet substructure, JHEP 06 (2013) 108 [arXiv: 1305.0007] [INSPIRE].

[82] J. Thaler and K. Van Tilburg, Identifying boosted objects with $N$-subjettiness, JHEP 03 (2011) 015 [arXiv: 1011.2268] [INSPIRE].

[83] A. Dominguez et al., CMS technical design report for the pixel detector upgrade, CERN-LHCC-2012-016 (2012) [INSPIRE].

[84] S. Bernstein, Démonstration du théorème de Weierstrass fondée sur le calcul des probabilitiés, Comm. Soc. Math. Kharkov 13 (1912) 1. 
[85] R.A. Fisher, On the interpretation of $\chi^{2}$ from contingency tables, and the calculation of $P$, J. Roy. Stat. Soc. 85 (1922) 87.

[86] CMS collaboration, Search for low mass vector resonances decaying into quark-antiquark pairs in proton-proton collisions at $\sqrt{s}=13$ TeV, Phys. Rev. D 100 (2019) 112007 [arXiv: 1909.04114] [INSPIRE].

[87] CMS collaboration, Identification of b-quark jets with the CMS experiment, 2013 JINST 8 P04013 [arXiv: 1211.4462] [INSPIRE].

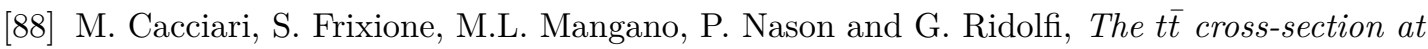
$1.8 \mathrm{TeV}$ and $1.96 \mathrm{TeV}$ : a study of the systematics due to parton densities and scale dependence, JHEP 04 (2004) 068 [hep-ph/0303085] [inSPIRE].

[89] S. Catani, D. de Florian, M. Grazzini and P. Nason, Soft gluon resummation for Higgs boson production at hadron colliders, JHEP 07 (2003) 028 [hep-ph/0306211] [INSPIRE].

[90] CMS collaboration, Search for new physics in final states with an energetic jet or a hadronically decaying $W$ or $Z$ boson and transverse momentum imbalance at $\sqrt{s}=13 \mathrm{TeV}$, Phys. Rev. D 97 (2018) 092005 [arXiv:1712.02345] [INSPIRE].

[91] A. Denner, S. Dittmaier, T. Kasprzik and A. Muck, Electroweak corrections to $W+$ jet hadroproduction including leptonic W-boson decays, JHEP 08 (2009) 075 [arXiv:0906.1656] [INSPIRE].

[92] A. Denner, S. Dittmaier, T. Kasprzik and A. Muck, Electroweak corrections to dilepton + jet production at hadron colliders, JHEP 06 (2011) 069 [arXiv:1103.0914] [INSPIRE].

[93] A. Denner, S. Dittmaier, T. Kasprzik and A. Mück, Electroweak corrections to monojet production at the LHC, Eur. Phys. J. C 73 (2013) 2297 [arXiv:1211.5078] [InSPIRE].

[94] J.H. Kühn, A. Kulesza, S. Pozzorini and M. Schulze, Electroweak corrections to hadronic photon production at large transverse momenta, JHEP 03 (2006) 059 [hep-ph/0508253] [INSPIRE].

[95] CMS collaboration, Determination of jet energy calibration and transverse momentum resolution in CMS, 2011 JINST 6 P11002 [arXiv:1107.4277] [INSPIRE].

[96] CMS collaboration, CMS luminosity measurements for the 2016 data taking period, CMS-PAS-LUM-17-001 (2017) [INSPIRE].

[97] ATLAS and CMS collaboration and the LHC Higgs Combination Group collaboration, Procedure for the LHC Higgs boson search combination in Summer 2011, CMS-NOTE-2011-005 (2011) [INSPIRE].

[98] CMS collaboration, Precise determination of the mass of the Higgs boson and tests of compatibility of its couplings with the standard model predictions using proton collisions at 7 and $8 \mathrm{TeV}$, Eur. Phys. J. C 75 (2015) 212 [arXiv:1412.8662] [InSPIRE].

[99] F.J. Massey Jr., The Kolmogorov-Smirnov test for goodness of fit, J. Am. Stat. Assoc. 46 (1951) 68.

[100] A.L. Read, Presentation of search results: the CL $L_{s}$ technique, J. Phys. G 28 (2002) 2693 [INSPIRE].

[101] T. Junk, Confidence level computation for combining searches with small statistics, Nucl. Instrum. Meth. A 434 (1999) 435 [hep-ex/9902006] [INSPIRE]. 
[102] G. Cowan, K. Cranmer, E. Gross and O. Vitells, Asymptotic formulae for likelihood-based tests of new physics, Eur. Phys. J. C 71 (2011) 1554 [Erratum ibid. 73 (2013) 2501] [arXiv: 1007.1727] [INSPIRE].

[103] L. Lyons, H.B. Prosper and A. De Roeck, eds., Statistical issues for LHC physics, in the proceedings of the Workshop PHYSTAT-LHC, June 27-29, Geneva, Switzerland (2017) [INSPIRE].

[104] N. Berger et al., Simplified template cross sections — Stage 1.1, arXiv:1906.02754 [INSPIRE].

[105] P.C. Hansen, The L-curve and its use in the numerical treatment of inverse problems, in Computational inverse problems in electrocardiology, P.R. Johnston ed., WIT Press, Southampton U.K. (2001). 


\section{The CMS collaboration}

Yerevan Physics Institute, Yerevan, Armenia

A.M. Sirunyan ${ }^{\dagger}$, A. Tumasyan

\section{Institut für Hochenergiephysik, Wien, Austria}

W. Adam, F. Ambrogi, T. Bergauer, M. Dragicevic, J. Erö, A. Escalante Del Valle, R. Frühwirth ${ }^{1}$, M. Jeitler ${ }^{1}$, N. Krammer, L. Lechner, D. Liko, T. Madlener, I. Mikulec, F.M. Pitters, N. Rad, J. Schieck ${ }^{1}$, R. Schöfbeck, M. Spanring, S. Templ, W. Waltenberger, C.-E. Wulz ${ }^{1}$, M. Zarucki

Institute for Nuclear Problems, Minsk, Belarus

V. Chekhovsky, A. Litomin, V. Makarenko, J. Suarez Gonzalez

Universiteit Antwerpen, Antwerpen, Belgium

M.R. Darwish ${ }^{2}$, E.A. De Wolf, D. Di Croce, X. Janssen, T. Kello ${ }^{3}$, A. Lelek, M. Pieters, H. Rejeb Sfar, H. Van Haevermaet, P. Van Mechelen, S. Van Putte, N. Van Remortel

\section{Vrije Universiteit Brussel, Brussel, Belgium}

F. Blekman, E.S. Bols, S.S. Chhibra, J. D'Hondt, J. De Clercq, D. Lontkovskyi, S. Lowette, I. Marchesini, S. Moortgat, A. Morton, Q. Python, S. Tavernier, W. Van Doninck, P. Van Mulders

\section{Université Libre de Bruxelles, Bruxelles, Belgium}

D. Beghin, B. Bilin, B. Clerbaux, G. De Lentdecker, H. Delannoy, B. Dorney, L. Favart, A. Grebenyuk, A.K. Kalsi, I. Makarenko, L. Moureaux, L. Pétré, A. Popov, N. Postiau, E. Starling, L. Thomas, C. Vander Velde, P. Vanlaer, D. Vannerom, L. Wezenbeek

\section{Ghent University, Ghent, Belgium}

T. Cornelis, D. Dobur, M. Gruchala, I. Khvastunov ${ }^{4}$, M. Niedziela, C. Roskas, K. Skovpen, M. Tytgat, W. Verbeke, B. Vermassen, M. Vit

\section{Université Catholique de Louvain, Louvain-la-Neuve, Belgium}

G. Bruno, F. Bury, C. Caputo, P. David, C. Delaere, M. Delcourt, I.S. Donertas, A. Giammanco, V. Lemaitre, K. Mondal, J. Prisciandaro, A. Taliercio, M. Teklishyn, P. Vischia, S. Wuyckens, J. Zobec

Centro Brasileiro de Pesquisas Fisicas, Rio de Janeiro, Brazil

G.A. Alves, G. Correia Silva, C. Hensel, A. Moraes

Universidade do Estado do Rio de Janeiro, Rio de Janeiro, Brazil

W.L. Aldá Júnior, E. Belchior Batista Das Chagas, H. BRANDAO MALBOUISSON, W. Carvalho, J. Chinellato ${ }^{5}$, E. Coelho, E.M. Da Costa, G.G. Da Silveira ${ }^{6}$, D. De Jesus Damiao, S. Fonseca De Souza, J. Martins ${ }^{7}$, D. Matos Figueiredo, M. Medina Jaime ${ }^{8}$, M. Melo De Almeida, C. Mora Herrera, L. Mundim, H. Nogima, P. Rebello Teles, L.J. Sanchez Rosas, A. Santoro, S.M. Silva Do Amaral, A. Sznajder, M. Thiel, E.J. Tonelli Manganote ${ }^{5}$, F. Torres Da Silva De Araujo, A. Vilela Pereira 
Universidade Estadual Paulista ${ }^{a}$, Universidade Federal do $\mathrm{ABC}^{b}$, São Paulo, Brazil

C.A. Bernardes ${ }^{a}$, L. Calligaris ${ }^{a}$, T.R. Fernandez Perez Tomei ${ }^{a}$, E.M. Gregores ${ }^{b}$, D.S. Lemos ${ }^{a}$, P.G. Mercadante ${ }^{b}$, S.F. Novaes ${ }^{a}$, Sandra S. Padula ${ }^{a}$

Institute for Nuclear Research and Nuclear Energy, Bulgarian Academy of Sciences, Sofia, Bulgaria

A. Aleksandrov, G. Antchev, I. Atanasov, R. Hadjiiska, P. Iaydjiev, M. Misheva,

M. Rodozov, M. Shopova, G. Sultanov

University of Sofia, Sofia, Bulgaria

M. Bonchev, A. Dimitrov, T. Ivanov, L. Litov, B. Pavlov, P. Petkov, A. Petrov

Beihang University, Beijing, China

W. Fang ${ }^{3}$, Q. Guo, H. Wang, L. Yuan

Department of Physics, Tsinghua University, Beijing, China

M. Ahmad, Z. Hu, Y. Wang

Institute of High Energy Physics, Beijing, China

E. Chapon, G.M. Chen ${ }^{9}$, H.S. Chen ${ }^{9}$, M. Chen, D. Leggat, H. Liao, Z. Liu, R. Sharma,

A. Spiezia, J. Tao, J. Thomas-wilsker, J. Wang, H. Zhang, S. Zhang ${ }^{9}$, J. Zhao

State Key Laboratory of Nuclear Physics and Technology, Peking University, Beijing, China

A. Agapitos, Y. Ban, C. Chen, A. Levin, J. Li, Q. Li, M. Lu, X. Lyu, Y. Mao, S.J. Qian,

D. Wang, Q. Wang, J. Xiao

Sun Yat-Sen University, Guangzhou, China

Z. You

Institute of Modern Physics and Key Laboratory of Nuclear Physics and Ionbeam Application (MOE) — Fudan University, Shanghai, China

X. $\mathrm{Gao}^{3}$

Zhejiang University, Hangzhou, China

M. Xiao

Universidad de Los Andes, Bogota, Colombia

C. Avila, A. Cabrera, C. Florez, J. Fraga, A. Sarkar, M.A. Segura Delgado

Universidad de Antioquia, Medellin, Colombia

J. Jaramillo, J. Mejia Guisao, F. Ramirez, J.D. Ruiz Alvarez, C.A. Salazar González,

N. Vanegas Arbelaez

University of Split, Faculty of Electrical Engineering, Mechanical Engineering and Naval Architecture, Split, Croatia

D. Giljanovic, N. Godinovic, D. Lelas, I. Puljak, T. Sculac 
University of Split, Faculty of Science, Split, Croatia

Z. Antunovic, M. Kovac

Institute Rudjer Boskovic, Zagreb, Croatia

V. Brigljevic, D. Ferencek, D. Majumder, B. Mesic, M. Roguljic, A. Starodumov ${ }^{10}$, T. Susa

University of Cyprus, Nicosia, Cyprus

M.W. Ather, A. Attikis, E. Erodotou, A. Ioannou, G. Kole, M. Kolosova, S. Konstantinou,

G. Mavromanolakis, J. Mousa, C. Nicolaou, F. Ptochos, P.A. Razis, H. Rykaczewski,

H. Saka, D. Tsiakkouri

Charles University, Prague, Czech Republic

M. Finger ${ }^{11}$, M. Finger Jr. ${ }^{11}$, A. Kveton, J. Tomsa

Escuela Politecnica Nacional, Quito, Ecuador

E. Ayala

Universidad San Francisco de Quito, Quito, Ecuador

E. Carrera Jarrin

Academy of Scientific Research and Technology of the Arab Republic of Egypt, Egyptian Network of High Energy Physics, Cairo, Egypt

H. Abdalla ${ }^{12}$, S. Khalil ${ }^{13}$, E. Salama ${ }^{14,15}$

Center for High Energy Physics (CHEP-FU), Fayoum University, El-Fayoum, Egypt

A. Lotfy, M.A. Mahmoud

National Institute of Chemical Physics and Biophysics, Tallinn, Estonia

S. Bhowmik, A. Carvalho Antunes De Oliveira, R.K. Dewanjee, K. Ehataht, M. Kadastik, M. Raidal, C. Veelken

Department of Physics, University of Helsinki, Helsinki, Finland

P. Eerola, L. Forthomme, H. Kirschenmann, K. Osterberg, M. Voutilainen

Helsinki Institute of Physics, Helsinki, Finland

E. Brücken, F. Garcia, J. Havukainen, V. Karimäki, M.S. Kim, R. Kinnunen, T. Lampén, K. Lassila-Perini, S. Laurila, S. Lehti, T. Lindén, H. Siikonen, E. Tuominen, J. Tuominiemi

Lappeenranta University of Technology, Lappeenranta, Finland

P. Luukka, T. Tuuva

IRFU, CEA, Université Paris-Saclay, Gif-sur-Yvette, France

M. Besancon, F. Couderc, M. Dejardin, D. Denegri, J.L. Faure, F. Ferri, S. Ganjour, A. Givernaud, P. Gras, G. Hamel de Monchenault, P. Jarry, B. Lenzi, E. Locci, J. Malcles, J. Rander, A. Rosowsky, M.Ö. Sahin, A. Savoy-Navarro ${ }^{16}$, M. Titov, G.B. Yu

Laboratoire Leprince-Ringuet, CNRS/IN2P3, Ecole Polytechnique, Institut Polytechnique de Paris, Paris, France

S. Ahuja, C. Amendola, F. Beaudette, M. Bonanomi, P. Busson, C. Charlot, O. Davignon, B. Diab, G. Falmagne, R. Granier de Cassagnac, I. Kucher, A. Lobanov, C. Martin Perez, 
M. Nguyen, C. Ochando, P. Paganini, J. Rembser, R. Salerno, J.B. Sauvan, Y. Sirois, A. Zabi, A. Zghiche

\section{Université de Strasbourg, CNRS, IPHC UMR 7178, Strasbourg, France}

J.-L. Agram ${ }^{17}$, J. Andrea, D. Bloch, G. Bourgatte, J.-M. Brom, E.C. Chabert, C. Collard, J.-C. Fontaine ${ }^{17}$, D. Gelé, U. Goerlach, C. Grimault, A.-C. Le Bihan, P. Van Hove

Université de Lyon, Université Claude Bernard Lyon 1, CNRS-IN2P3, Institut de Physique Nucléaire de Lyon, Villeurbanne, France

E. Asilar, S. Beauceron, C. Bernet, G. Boudoul, C. Camen, A. Carle, N. Chanon, D. Contardo, P. Depasse, H. El Mamouni, J. Fay, S. Gascon, M. Gouzevitch, B. Ille, Sa. Jain, I.B. Laktineh, H. Lattaud, A. Lesauvage, M. Lethuillier, L. Mirabito, L. Torterotot, G. Touquet, M. Vander Donckt, S. Viret

\section{Georgian Technical University, Tbilisi, Georgia}

A. Khvedelidze ${ }^{11}$, Z. Tsamalaidze ${ }^{11}$

\section{RWTH Aachen University, I. Physikalisches Institut, Aachen, Germany}

L. Feld, K. Klein, M. Lipinski, D. Meuser, A. Pauls, M. Preuten, M.P. Rauch, J. Schulz, M. Teroerde

\section{RWTH Aachen University, III. Physikalisches Institut A, Aachen, Germany}

D. Eliseev, M. Erdmann, P. Fackeldey, B. Fischer, S. Ghosh, T. Hebbeker, K. Hoepfner, H. Keller, L. Mastrolorenzo, M. Merschmeyer, A. Meyer, P. Millet, G. Mocellin, S. Mondal, S. Mukherjee, D. Noll, A. Novak, T. Pook, A. Pozdnyakov, T. Quast, M. Radziej, Y. Rath, H. Reithler, J. Roemer, A. Schmidt, S.C. Schuler, A. Sharma, S. Wiedenbeck, S. Zaleski

RWTH Aachen University, III. Physikalisches Institut B, Aachen, Germany

C. Dziwok, G. Flügge, W. Haj Ahmad ${ }^{18}$, O. Hlushchenko, T. Kress, A. Nowack, C. Pistone, O. Pooth, D. Roy, H. Sert, A. Stahl ${ }^{19}$, T. Ziemons

\section{Deutsches Elektronen-Synchrotron, Hamburg, Germany}

H. Aarup Petersen, M. Aldaya Martin, P. Asmuss, I. Babounikau, S. Baxter, O. Behnke, A. Bermúdez Martínez, A.A. Bin Anuar, K. Borras ${ }^{20}$, V. Botta, D. Brunner, A. Campbell, A. Cardini, P. Connor, S. Consuegra Rodríguez, V. Danilov, A. De Wit, M.M. Defranchis, L. Didukh, D. Domínguez Damiani, G. Eckerlin, D. Eckstein, T. Eichhorn, A. Elwood, L.I. Estevez Banos, E. Gallo ${ }^{21}$, A. Geiser, A. Giraldi, A. Grohsjean, M. Guthoff, A. Harb, A. Jafari ${ }^{22}$, N.Z. Jomhari, H. Jung, A. Kasem ${ }^{20}$, M. Kasemann, H. Kaveh, C. Kleinwort, J. Knolle, D. Krücker, W. Lange, T. Lenz, J. Lidrych, K. Lipka, W. Lohmann ${ }^{23}$, R. Mankel, I.-A. Melzer-Pellmann, J. Metwally, A.B. Meyer, M. Meyer, M. Missiroli, J. Mnich, A. Mussgiller, V. Myronenko, Y. Otarid, D. Pérez Adán, S.K. Pflitsch, D. Pitzl, A. Raspereza, A. Saggio, A. Saibel, M. Savitskyi, V. Scheurer, P. Schütze, C. Schwanenberger, R. Shevchenko, A. Singh, R.E. Sosa Ricardo, H. Tholen, N. Tonon, O. Turkot, A. Vagnerini, M. Van De Klundert, R. Walsh, D. Walter, Y. Wen, K. Wichmann, C. Wissing, S. Wuchterl, O. Zenaiev, R. Zlebcik 


\section{University of Hamburg, Hamburg, Germany}

R. Aggleton, S. Bein, L. Benato, A. Benecke, K. De Leo, T. Dreyer, A. Ebrahimi, F. Feindt, A. Fröhlich, C. Garbers, E. Garutti, P. Gunnellini, J. Haller, A. Hinzmann, A. Karavdina, G. Kasieczka, R. Klanner, R. Kogler, V. Kutzner, J. Lange, T. Lange, A. Malara, J. Multhaup, C.E.N. Niemeyer, A. Nigamova, K.J. Pena Rodriguez, O. Rieger, P. Schleper, S. Schumann, J. Schwandt, D. Schwarz, J. Sonneveld, H. Stadie, G. Steinbrück, B. Vormwald, I. Zoi

\section{Karlsruher Institut fuer Technologie, Karlsruhe, Germany}

M. Baselga, S. Baur, J. Bechtel, T. Berger, E. Butz, R. Caspart, T. Chwalek, W. De Boer, A. Dierlamm, A. Droll, K. El Morabit, N. Faltermann, K. Flöh, M. Giffels, A. Gottmann, F. Hartmann ${ }^{19}$, C. Heidecker, U. Husemann, M.A. Iqbal, I. Katkov ${ }^{24}$, P. Keicher, R. Koppenhöfer, S. Maier, M. Metzler, S. Mitra, M.U. Mozer, D. Müller, Th. Müller, M. Musich, G. Quast, K. Rabbertz, J. Rauser, D. Savoiu, D. Schäfer, M. Schnepf, M. Schröder, D. Seith, I. Shvetsov, H.J. Simonis, R. Ulrich, M. Wassmer, M. Weber, C. Wöhrmann, R. Wolf, S. Wozniewski

Institute of Nuclear and Particle Physics (INPP), NCSR Demokritos, Aghia Paraskevi, Greece

G. Anagnostou, P. Asenov, G. Daskalakis, T. Geralis, A. Kyriakis, D. Loukas, G. Paspalaki, A. Stakia

\section{National and Kapodistrian University of Athens, Athens, Greece}

M. Diamantopoulou, D. Karasavvas, G. Karathanasis, P. Kontaxakis, C.K. Koraka, A. Manousakis-katsikakis, A. Panagiotou, I. Papavergou, N. Saoulidou, K. Theofilatos, K. Vellidis, E. Vourliotis

National Technical University of Athens, Athens, Greece

G. Bakas, K. Kousouris, I. Papakrivopoulos, G. Tsipolitis, A. Zacharopoulou

University of Ioánnina, Ioánnina, Greece

I. Evangelou, C. Foudas, P. Gianneios, P. Katsoulis, P. Kokkas, S. Mallios, K. Manitara, N. Manthos, I. Papadopoulos, J. Strologas

MTA-ELTE Lendület CMS Particle and Nuclear Physics Group, Eötvös Loránd University, Budapest, Hungary

M. Bartók ${ }^{25}$, R. Chudasama, M. Csanad, M.M.A. Gadallah ${ }^{26}$, S. Lökös ${ }^{27}$, P. Major, K. Mandal, A. Mehta, G. Pasztor, O. Surányi, G.I. Veres

Wigner Research Centre for Physics, Budapest, Hungary

G. Bencze, C. Hajdu, D. Horvath ${ }^{28}$, F. Sikler, V. Veszpremi, G. Vesztergombi ${ }^{\dagger}$

Institute of Nuclear Research ATOMKI, Debrecen, Hungary

S. Czellar, J. Karancsi ${ }^{25}$, J. Molnar, Z. Szillasi, D. Teyssier

Institute of Physics, University of Debrecen, Debrecen, Hungary

P. Raics, Z.L. Trocsanyi, B. Ujvari 
Eszterhazy Karoly University, Karoly Robert Campus, Gyongyos, Hungary

T. Csorgo, F. Nemes, T. Novak

Indian Institute of Science (IISc), Bangalore, India

S. Choudhury, J.R. Komaragiri, D. Kumar, L. Panwar, P.C. Tiwari

National Institute of Science Education and Research, HBNI, Bhubaneswar, India

S. Bahinipati ${ }^{29}$, D. Dash, C. Kar, P. Mal, T. Mishra, V.K. Muraleedharan Nair Bindhu, A. Nayak ${ }^{30}$, D.K. Sahoo $^{29}$, N. Sur, S.K. Swain

Panjab University, Chandigarh, India

S. Bansal, S.B. Beri, V. Bhatnagar, S. Chauhan, N. Dhingra ${ }^{31}$, R. Gupta, A. Kaur, S. Kaur, P. Kumari, M. Lohan, M. Meena, K. Sandeep, S. Sharma, J.B. Singh, A.K. Virdi

University of Delhi, Delhi, India

A. Ahmed, A. Bhardwaj, B.C. Choudhary, R.B. Garg, M. Gola, S. Keshri, A. Kumar, M. Naimuddin, P. Priyanka, K. Ranjan, A. Shah

Saha Institute of Nuclear Physics, HBNI, Kolkata, India

M. Bharti ${ }^{32}$, R. Bhattacharya, S. Bhattacharya, D. Bhowmik, S. Dutta, S. Ghosh,

B. Gomber ${ }^{33}$, M. Maity ${ }^{34}$, S. Nandan, P. Palit, A. Purohit, P.K. Rout, G. Saha, S. Sarkar, M. Sharan, B. Singh ${ }^{32}$, S. Thakur ${ }^{32}$

Indian Institute of Technology Madras, Madras, India

P.K. Behera, S.C. Behera, P. Kalbhor, A. Muhammad, R. Pradhan, P.R. Pujahari, A. Sharma, A.K. Sikdar

Bhabha Atomic Research Centre, Mumbai, India

D. Dutta, V. Jha, V. Kumar, D.K. Mishra, K. Naskar ${ }^{35}$, P.K. Netrakanti, L.M. Pant, P. Shukla

Tata Institute of Fundamental Research-A, Mumbai, India

T. Aziz, M.A. Bhat, S. Dugad, R. Kumar Verma, U. Sarkar

Tata Institute of Fundamental Research-B, Mumbai, India

S. Banerjee, S. Bhattacharya, S. Chatterjee, P. Das, M. Guchait, S. Karmakar, S. Kumar, G. Majumder, K. Mazumdar, S. Mukherjee, D. Roy, N. Sahoo

Indian Institute of Science Education and Research (IISER), Pune, India

S. Dube, B. Kansal, A. Kapoor, K. Kothekar, S. Pandey, A. Rane, A. Rastogi, S. Sharma

Department of Physics, Isfahan University of Technology, Isfahan, Iran

H. Bakhshiansohi ${ }^{36}$

Institute for Research in Fundamental Sciences (IPM), Tehran, Iran

S. Chenarani ${ }^{37}$, S.M. Etesami, M. Khakzad, M. Mohammadi Najafabadi

University College Dublin, Dublin, Ireland

M. Felcini, M. Grunewald 
INFN Sezione di Bari ${ }^{a}$, Università di $\mathrm{Bari}^{b}$, Politecnico di Bari ${ }^{c}$, Bari, Italy

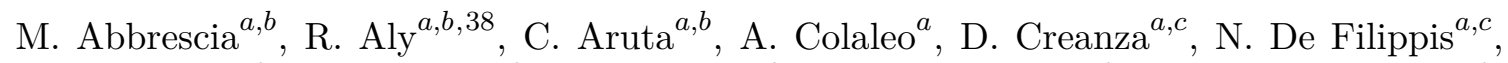
M. De Palma ${ }^{a, b}$, A. Di Florio ${ }^{a, b}$, A. Di Pilato ${ }^{a, b}$, W. Elmetenawee ${ }^{a, b}$, L. Fiore $^{a}$, A. Gelmi $^{a, b}$,

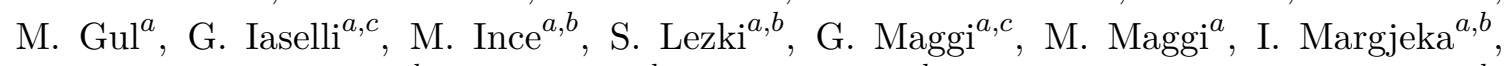
J.A. Merlin ${ }^{a}$, S. My ${ }^{a, b}$, S. Nuzzo ${ }^{a, b}$, A. Pompili ${ }^{a, b}$, G. Pugliese ${ }^{a, c}$, G. Selvaggi ${ }^{a, b}$, L. Silvestris ${ }^{a}$, F.M. Simone ${ }^{a, b}$, R. Venditti ${ }^{a}$, P. Verwilligen $^{a}$

INFN Sezione di Bologna ${ }^{a}$, Università di Bologna ${ }^{b}$, Bologna, Italy

G. Abbiendi ${ }^{a}$, C. Battilana ${ }^{a, b}$, D. Bonacorsi ${ }^{a, b}$, L. Borgonovi $^{a, b}$, S. Braibant-Giacomelli $^{a, b}$, L. Brigliadori ${ }^{a, b}$, R. Campanini ${ }^{a, b}$, P. Capiluppi ${ }^{a, b}$, A. Castro $^{a, b}$, F.R. Cavallo $^{a}$, C. Ciocca $^{a}$, M. Cuffiani ${ }^{a, b}$, G.M. Dallavalle ${ }^{a}$, T. Diotalevi ${ }^{a, b}$, F. Fabbri $^{a}$, A. Fanfani ${ }^{a, b}$, E. Fontanesi $^{a, b}{ }^{,}$ P. Giacomelli ${ }^{a}$, C. Grandi ${ }^{a}$, L. Guiducci ${ }^{a, b}$, F. Iemmi ${ }^{a, b}$, S. Lo Meo ${ }^{a, 39}$, S. Marcellini ${ }^{a}$,

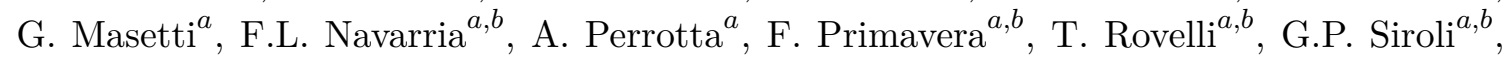
N. $\operatorname{Tosi}^{a}$

INFN Sezione di Catania ${ }^{a}$, Università di Catania ${ }^{b}$, Catania, Italy

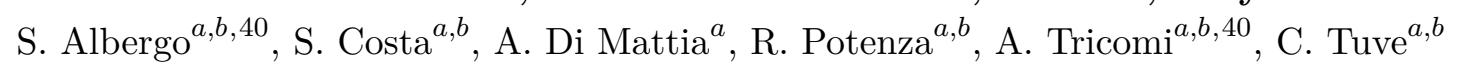

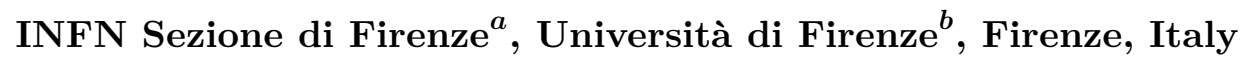

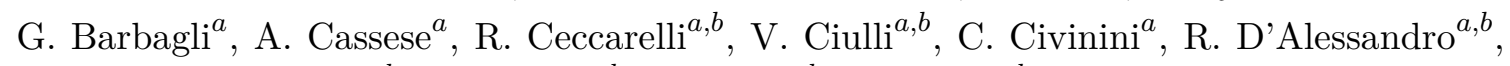

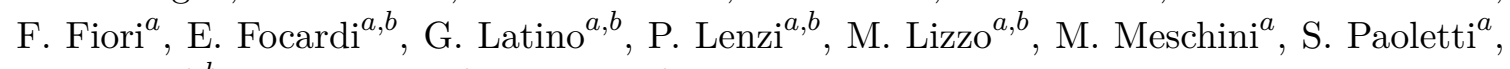
R. Seidita ${ }^{a, b}$, G. Sguazzoni ${ }^{a}$, L. Viliani $^{a}$

INFN Laboratori Nazionali di Frascati, Frascati, Italy

L. Benussi, S. Bianco, D. Piccolo

INFN Sezione di Genova ${ }^{a}$, Università di Genova ${ }^{b}$, Genova, Italy

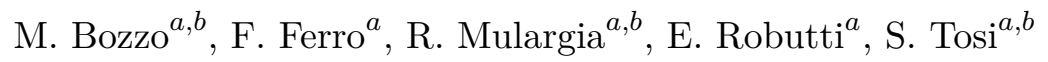

INFN Sezione di Milano-Bicocca $^{a}$, Università di Milano-Bicocca ${ }^{b}$, Milano, Italy

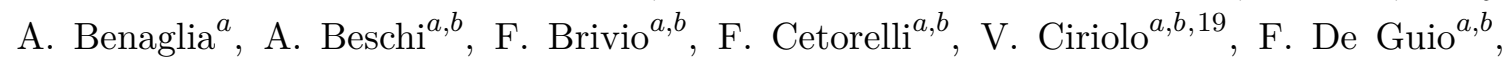
M.E. Dinardo ${ }^{a, b}$, P. Dini $^{a}$, S. Gennai ${ }^{a}$, A. Ghezzi ${ }^{a, b}$, P. Govoni $^{a, b}$, L. Guzzi $^{a, b}$, M. Malberti $^{a}$, S. Malvezzi ${ }^{a}$, D. Menasce ${ }^{a}$, F. Monti ${ }^{a, b}$, L. Moroni ${ }^{a}$, M. Paganoni ${ }^{a, b}$, D. Pedrini ${ }^{a}$, S. Ragazzi ${ }^{a, b}$, T. Tabarelli de Fatis ${ }^{a, b}$, D. Valsecchi ${ }^{a, b, 19}$, D. Zuolo ${ }^{a, b}$

INFN Sezione di Napoli ${ }^{a}$, Università di Napoli 'Federico II'b , Napoli, Italy, Università della Basilicata $^{c}$, Potenza, Italy, Università G. Marconi ${ }^{d}$, Roma, Italy

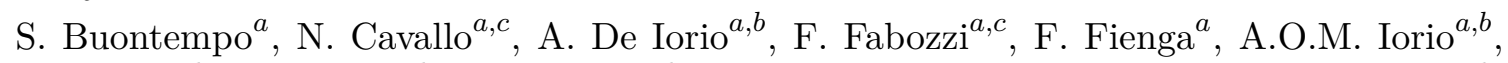
L. Layer ${ }^{a, b}$, L. Lista ${ }^{a, b}$, S. Meola ${ }^{a, d, 19}$, P. Paolucci ${ }^{a, 19}$, B. Rossi ${ }^{a}$, C. Sciacca ${ }^{a, b}$, E. Voevodina ${ }^{a, b}$

INFN Sezione di Padova ${ }^{a}$, Università di Padova ${ }^{b}$, Padova, Italy, Università di Trento $^{c}$, Trento, Italy

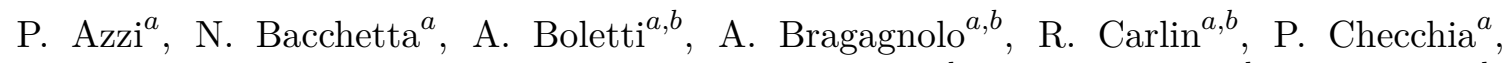
P. De Castro Manzano ${ }^{a}$, T. Dorigo ${ }^{a}$, F. Gasparini ${ }^{a, b}$, U. Gasparini ${ }^{a, b}$, S.Y. Hoh ${ }^{a, b}$, M. Margoni ${ }^{a, b}$, A.T. Meneguzzo ${ }^{a, b}$, M. Presilla ${ }^{b}$, P. Ronchese ${ }^{a, b}$, R. Rossin ${ }^{a, b}$, 
F. Simonetto ${ }^{a, b}$, G. Strong, A. Tiko ${ }^{a}$, M. Tosi ${ }^{a, b}$, H. YARAR $^{a, b}$, M. Zanetti $^{a, b}$, P. Zotto $^{a, b}$, A. Zucchetta ${ }^{a, b}$, G. Zumerle ${ }^{a, b}$

INFN Sezione di Pavia ${ }^{a}$, Università di Pavia ${ }^{b}$, Pavia, Italy

A. Braghieri ${ }^{a}$, S. Calzaferri ${ }^{a, b}$, D. Fiorina ${ }^{a, b}$, P. Montagna ${ }^{a, b}$, S.P. Ratti ${ }^{a, b}$, V. Re ${ }^{a}$,

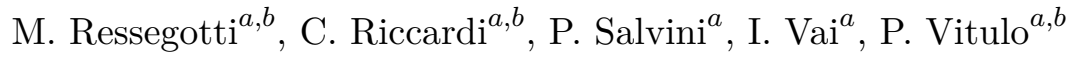

INFN Sezione di Perugia ${ }^{a}$, Università di Perugia $^{b}$, Perugia, Italy

M. Biasini ${ }^{a, b}$, G.M. Bilei ${ }^{a}$, D. Ciangottini ${ }^{a, b}$, L. Fanò ${ }^{a, b}$, P. Lariccia ${ }^{a, b}$ G. Mantovani $^{a, b}{ }^{a}$

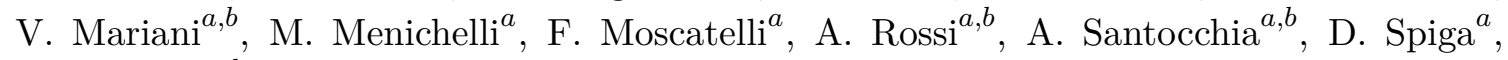
T. Tedeschi ${ }^{a, b}$

INFN Sezione di Pisa ${ }^{a}$, Università di Pisa ${ }^{b}$, Scuola Normale Superiore di $\mathrm{Pisa}^{c}$, Pisa, Italy

K. Androsov $^{a}$, P. Azzurri ${ }^{a}$, G. Bagliesi ${ }^{a}$, V. Bertacchi ${ }^{a, c}$, L. Bianchini $^{a}$, T. Boccali ${ }^{a}$, R. Castaldi ${ }^{a}$, M.A. Ciocci ${ }^{a, b}$, R. Dell'Orso ${ }^{a}$, M.R. Di Domenico ${ }^{a, b}$, S. Donato ${ }^{a}$,

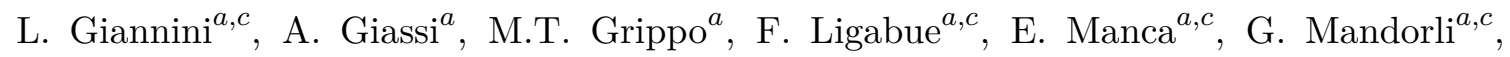
A. Messineo ${ }^{a, b}$, F. Palla ${ }^{a}$, G. Ramirez-Sanchez ${ }^{a, c}$, A. Rizzi ${ }^{a, b}$, G. Rolandi ${ }^{a, c}$, S. Roy Chowdhury ${ }^{a, c}$, A. Scribano ${ }^{a}$, N. Shafiei ${ }^{a, b}$, P. Spagnolo $^{a}$, R. Tenchini ${ }^{a}$, G. Tonelli $^{a, b}$,

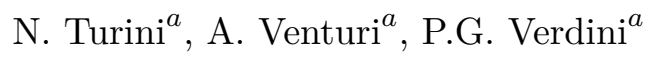

INFN Sezione di Roma ${ }^{a}$, Sapienza Università di Roma ${ }^{b}$, Rome, Italy

F. Cavallari ${ }^{a}$, M. Cipriani ${ }^{a, b}$, D. Del Re ${ }^{a, b}$, E. Di $\operatorname{Marco}^{a}$, M. Diemoz $^{a}$, E. Longo ${ }^{a, b}$, P. Meridiani ${ }^{a}$, G. Organtini ${ }^{a, b}$, F. Pandolfi ${ }^{a}$, R. Paramatti ${ }^{a, b}$, C. Quaranta ${ }^{a, b}$,

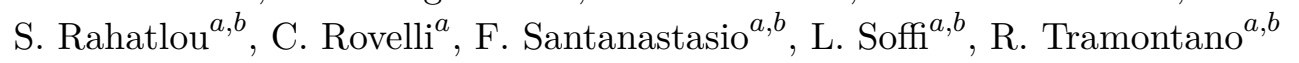

INFN Sezione di Torino ${ }^{a}$, Università di Torino ${ }^{b}$, Torino, Italy, Università del Piemonte Orientale ${ }^{c}$, Novara, Italy

N. Amapane ${ }^{a, b}$, R. Arcidiacono ${ }^{a, c}$, S. Argiro ${ }^{a, b}$, M. Arneodo $^{a, c}$, N. Bartosik ${ }^{a}$, R. Bellan $^{a, b}$, A. Bellora ${ }^{a, b}$, C. Biino ${ }^{a}$, A. Cappati ${ }^{a, b}$, N. $\operatorname{Cartiglia}^{a}$, S. $\operatorname{Cometti}^{a}$, M. $\operatorname{Costa}^{a, b}$, R. Covarelli ${ }^{a, b}$, N. Demaria ${ }^{a}$, B. Kiani ${ }^{a, b}$, F. Legger ${ }^{a}$, C. Mariotti ${ }^{a}$, S. Maselli ${ }^{a}$, E. Migliore $^{a, b}$, V. Monaco ${ }^{a, b}$, E. Monteil ${ }^{a, b}$, M. Monteno $^{a}$, M.M. Obertino $^{a, b}$, G. Ortona $^{a}$, L. Pacher ${ }^{a, b}$, N. Pastrone ${ }^{a}$, M. Pelliccioni ${ }^{a}$, G.L. Pinna Angioni ${ }^{a, b}$, M. Ruspa ${ }^{a, c}$,

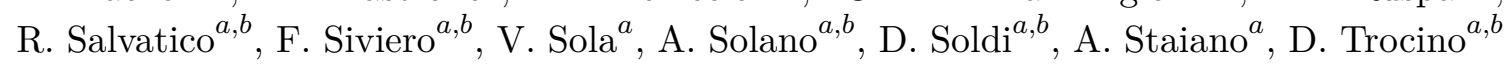

INFN Sezione di Trieste ${ }^{a}$, Università di Trieste ${ }^{b}$, Trieste, Italy

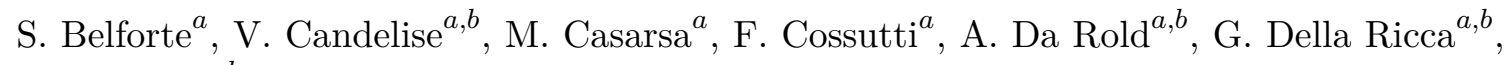
F. Vazzoler ${ }^{a, b}$

Kyungpook National University, Daegu, Korea

S. Dogra, C. Huh, B. Kim, D.H. Kim, G.N. Kim, J. Lee, S.W. Lee, C.S. Moon, Y.D. Oh, S.I. Pak, S. Sekmen, Y.C. Yang

Chonnam National University, Institute for Universe and Elementary Particles, Kwangju, Korea

H. Kim, D.H. Moon 
Hanyang University, Seoul, Korea

B. Francois, T.J. Kim, J. Park

Korea University, Seoul, Korea

S. Cho, S. Choi, Y. Go, S. Ha, B. Hong, K. Lee, K.S. Lee, J. Lim, J. Park, S.K. Park, J. Yoo

Kyung Hee University, Department of Physics, Seoul, Republic of Korea

J. Goh, A. Gurtu

Sejong University, Seoul, Korea

H.S. Kim, Y. Kim

Seoul National University, Seoul, Korea

J. Almond, J.H. Bhyun, J. Choi, S. Jeon, J. Kim, J.S. Kim, S. Ko, H. Kwon, H. Lee, K. Lee,

S. Lee, K. Nam, B.H. Oh, M. Oh, S.B. Oh, B.C. Radburn-Smith, H. Seo, U.K. Yang, I. Yoon

University of Seoul, Seoul, Korea

D. Jeon, J.H. Kim, B. Ko, J.S.H. Lee, I.C. Park, Y. Roh, D. Song, I.J. Watson

Yonsei University, Department of Physics, Seoul, Korea

H.D. Yoo

Sungkyunkwan University, Suwon, Korea

Y. Choi, C. Hwang, Y. Jeong, H. Lee, Y. Lee, I. Yu

Riga Technical University, Riga, Latvia

V. Veckalns ${ }^{41}$

Vilnius University, Vilnius, Lithuania

A. Juodagalvis, A. Rinkevicius, G. Tamulaitis

National Centre for Particle Physics, Universiti Malaya, Kuala Lumpur, Malaysia

W.A.T. Wan Abdullah, M.N. Yusli, Z. Zolkapli

Universidad de Sonora (UNISON), Hermosillo, Mexico

J.F. Benitez, A. Castaneda Hernandez, J.A. Murillo Quijada, L. Valencia Palomo

Centro de Investigacion y de Estudios Avanzados del IPN, Mexico City, Mexico

H. Castilla-Valdez, E. De La Cruz-Burelo, I. Heredia-De La Cruz ${ }^{42}$, R. Lopez-Fernandez,

A. Sanchez-Hernandez

Universidad Iberoamericana, Mexico City, Mexico

S. Carrillo Moreno, C. Oropeza Barrera, M. Ramirez-Garcia, F. Vazquez Valencia

Benemerita Universidad Autonoma de Puebla, Puebla, Mexico

J. Eysermans, I. Pedraza, H.A. Salazar Ibarguen, C. Uribe Estrada

Universidad Autónoma de San Luis Potosí, San Luis Potosí, Mexico

A. Morelos Pineda 
University of Montenegro, Podgorica, Montenegro

J. Mijuskovic ${ }^{4}$, N. Raicevic

University of Auckland, Auckland, New Zealand

D. Krofcheck

University of Canterbury, Christchurch, New Zealand

S. Bheesette, P.H. Butler

National Centre for Physics, Quaid-I-Azam University, Islamabad, Pakistan

A. Ahmad, M.I. Asghar, M.I.M. Awan, Q. Hassan, H.R. Hoorani, W.A. Khan, M.A. Shah, M. Shoaib, M. Waqas

AGH University of Science and Technology Faculty of Computer Science, Electronics and Telecommunications, Krakow, Poland

V. Avati, L. Grzanka, M. Malawski

National Centre for Nuclear Research, Swierk, Poland

H. Bialkowska, M. Bluj, B. Boimska, T. Frueboes, M. Górski, M. Kazana, M. Szleper, P. Traczyk, P. Zalewski

Institute of Experimental Physics, Faculty of Physics, University of Warsaw, Warsaw, Poland

K. Bunkowski, A. Byszuk ${ }^{43}$, K. Doroba, A. Kalinowski, M. Konecki, J. Krolikowski, M. Olszewski, M. Walczak

Laboratório de Instrumentação e Física Experimental de Partículas, Lisboa, Portugal

M. Araujo, P. Bargassa, D. Bastos, P. Faccioli, M. Gallinaro, J. Hollar, N. Leonardo, T. Niknejad, J. Seixas, K. Shchelina, O. Toldaiev, J. Varela

Joint Institute for Nuclear Research, Dubna, Russia

S. Afanasiev, P. Bunin, M. Gavrilenko, I. Golutvin, I. Gorbunov, A. Kamenev, V. Karjavine, A. Lanev, A. Malakhov, V. Matveev ${ }^{44,45}$, P. Moisenz, V. Palichik, V. Perelygin, M. Savina, D. Seitova, V. Shalaev, S. Shmatov, S. Shulha, V. Smirnov, O. Teryaev, N. Voytishin, A. Zarubin, I. Zhizhin

Petersburg Nuclear Physics Institute, Gatchina (St. Petersburg), Russia

G. Gavrilov, V. Golovtcov, Y. Ivanov, V. Kim ${ }^{46}$, E. Kuznetsova ${ }^{47}$, V. Murzin, V. Oreshkin, I. Smirnov, D. Sosnov, V. Sulimov, L. Uvarov, S. Volkov, A. Vorobyev

Institute for Nuclear Research, Moscow, Russia

Yu. Andreev, A. Dermenev, S. Gninenko, N. Golubev, A. Karneyeu, M. Kirsanov, N. Krasnikov, A. Pashenkov, G. Pivovarov, D. Tlisov, A. Toropin

Institute for Theoretical and Experimental Physics named by A.I. Alikhanov of NRC 'Kurchatov Institute', Moscow, Russia

V. Epshteyn, V. Gavrilov, N. Lychkovskaya, A. Nikitenko ${ }^{48}$, V. Popov, I. Pozdnyakov, G. Safronov, A. Spiridonov, A. Stepennov, M. Toms, E. Vlasov, A. Zhokin 
Moscow Institute of Physics and Technology, Moscow, Russia

T. Aushev

National Research Nuclear University 'Moscow Engineering Physics Institute' (MEPhI), Moscow, Russia

O. Bychkova, M. Chadeeva ${ }^{49}$, D. Philippov, E. Popova, V. Rusinov

P.N. Lebedev Physical Institute, Moscow, Russia

V. Andreev, M. Azarkin, I. Dremin, M. Kirakosyan, A. Terkulov

Skobeltsyn Institute of Nuclear Physics, Lomonosov Moscow State University, Moscow, Russia

A. Baskakov, A. Belyaev, E. Boos, V. Bunichev, M. Dubinin ${ }^{50}$, L. Dudko, A. Ershov, V. Klyukhin, O. Kodolova, I. Lokhtin, S. Obraztsov, M. Perfilov, V. Savrin

Novosibirsk State University (NSU), Novosibirsk, Russia

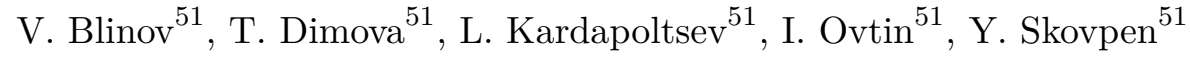

Institute for High Energy Physics of National Research Centre 'Kurchatov Institute', Protvino, Russia

I. Azhgirey, I. Bayshev, V. Kachanov, A. Kalinin, D. Konstantinov, V. Petrov, R. Ryutin, A. Sobol, S. Troshin, N. Tyurin, A. Uzunian, A. Volkov

National Research Tomsk Polytechnic University, Tomsk, Russia

A. Babaev, A. Iuzhakov, V. Okhotnikov, L. Sukhikh

Tomsk State University, Tomsk, Russia

V. Borchsh, V. Ivanchenko, E. Tcherniaev

University of Belgrade: Faculty of Physics and VINCA Institute of Nuclear Sciences, Belgrade, Serbia

P. Adzic ${ }^{52}$, P. Cirkovic, M. Dordevic, P. Milenovic, J. Milosevic

Centro de Investigaciones Energéticas Medioambientales y Tecnológicas (CIEMAT), Madrid, Spain

M. Aguilar-Benitez, J. Alcaraz Maestre, A. Álvarez Fernández, I. Bachiller, M. Barrio Luna, Cristina F. Bedoya, J.A. Brochero Cifuentes, C.A. Carrillo Montoya, M. Cepeda, M. Cerrada, N. Colino, B. De La Cruz, A. Delgado Peris, J.P. Fernández Ramos, J. Flix, M.C. Fouz, A. García Alonso, O. Gonzalez Lopez, S. Goy Lopez, J.M. Hernandez, M.I. Josa, D. Moran, Á. Navarro Tobar, A. Pérez-Calero Yzquierdo, J. Puerta Pelayo, I. Redondo, L. Romero, S. Sánchez Navas, M.S. Soares, A. Triossi, C. Willmott

Universidad Autónoma de Madrid, Madrid, Spain

C. Albajar, J.F. de Trocóniz, R. Reyes-Almanza

Universidad de Oviedo, Instituto Universitario de Ciencias y Tecnologías Espaciales de Asturias (ICTEA), Oviedo, Spain

B. Alvarez Gonzalez, J. Cuevas, C. Erice, J. Fernandez Menendez, S. Folgueras, I. Gonzalez Caballero, E. Palencia Cortezon, C. Ramón Álvarez, V. Rodríguez Bouza, S. Sanchez Cruz, A. Trapote 
Instituto de Física de Cantabria (IFCA), CSIC-Universidad de Cantabria, Santander, Spain

I.J. Cabrillo, A. Calderon, B. Chazin Quero, J. Duarte Campderros, M. Fernandez, P.J. Fernández Manteca, G. Gomez, C. Martinez Rivero, P. Martinez Ruiz del Arbol, F. Matorras, J. Piedra Gomez, C. Prieels, F. Ricci-Tam, T. Rodrigo, A. Ruiz-Jimeno, L. Russo ${ }^{53}$, L. Scodellaro, I. Vila, J.M. Vizan Garcia

University of Colombo, Colombo, Sri Lanka

MK Jayananda, B. Kailasapathy ${ }^{54}$, D.U.J. Sonnadara, DDC Wickramarathna

University of Ruhuna, Department of Physics, Matara, Sri Lanka

W.G.D. Dharmaratna, K. Liyanage, N. Perera, N. Wickramage

CERN, European Organization for Nuclear Research, Geneva, Switzerland

T.K. Aarrestad, D. Abbaneo, B. Akgun, E. Auffray, G. Auzinger, J. Baechler, P. Baillon, A.H. Ball, D. Barney, J. Bendavid, N. Beni, M. Bianco, A. Bocci, P. Bortignon, E. Bossini, E. Brondolin, T. Camporesi, G. Cerminara, L. Cristella, D. d'Enterria, A. Dabrowski, N. Daci, V. Daponte, A. David, A. De Roeck, M. Deile, R. Di Maria, M. Dobson, M. Dünser, N. Dupont, A. Elliott-Peisert, N. Emriskova, F. Fallavollita ${ }^{55}$, D. Fasanella, S. Fiorendi, G. Franzoni, J. Fulcher, W. Funk, S. Giani, D. Gigi, K. Gill, F. Glege, L. Gouskos, M. Guilbaud, D. Gulhan, M. Haranko, J. Hegeman, Y. Iiyama, V. Innocente, T. James, P. Janot, J. Kaspar, J. Kieseler, M. Komm, N. Kratochwil, C. Lange, P. Lecoq, K. Long, C. Lourenço, L. Malgeri, M. Mannelli, A. Massironi, F. Meijers, S. Mersi, E. Meschi, F. Moortgat, M. Mulders, J. Ngadiuba, J. Niedziela, S. Orfanelli, L. Orsini, F. Pantaleo ${ }^{19}$, L. Pape, E. Perez, M. Peruzzi, A. Petrilli, G. Petrucciani, A. Pfeiffer, M. Pierini, D. Rabady, A. Racz, M. Rieger, M. Rovere, H. Sakulin, J. Salfeld-Nebgen, S. Scarfi, C. Schäfer, C. Schwick, M. Selvaggi, A. Sharma, P. Silva, W. Snoeys, P. Sphicas ${ }^{56}$, J. Steggemann, S. Summers, V.R. Tavolaro, D. Treille, A. Tsirou, G.P. Van Onsem, A. Vartak, M. Verzetti, K.A. Wozniak, W.D. Zeuner

Paul Scherrer Institut, Villigen, Switzerland

L. Caminada ${ }^{57}$, W. Erdmann, R. Horisberger, Q. Ingram, H.C. Kaestli, D. Kotlinski, U. Langenegger, T. Rohe

ETH Zurich - Institute for Particle Physics and Astrophysics (IPA), Zurich, Switzerland

M. Backhaus, P. Berger, A. Calandri, N. Chernyavskaya, G. Dissertori, M. Dittmar, M. Donegà, C. Dorfer, T. Gadek, T.A. Gómez Espinosa, C. Grab, D. Hits, W. Lustermann, A.-M. Lyon, R.A. Manzoni, M.T. Meinhard, F. Micheli, F. Nessi-Tedaldi, F. Pauss, V. Perovic, G. Perrin, L. Perrozzi, S. Pigazzini, M.G. Ratti, M. Reichmann, C. Reissel, T. Reitenspiess, B. Ristic, D. Ruini, D.A. Sanz Becerra, M. Schönenberger, L. Shchutska, V. Stampf, M.L. Vesterbacka Olsson, R. Wallny, D.H. Zhu

Universität Zürich, Zurich, Switzerland

C. Amsler ${ }^{58}$, C. Botta, D. Brzhechko, M.F. Canelli, A. De Cosa, R. Del Burgo, J.K. Heikkilä, M. Huwiler, A. Jofrehei, B. Kilminster, S. Leontsinis, A. Macchiolo, 
P. Meiring, V.M. Mikuni, U. Molinatti, I. Neutelings, G. Rauco, A. Reimers, P. Robmann, K. Schweiger, Y. Takahashi, S. Wertz

National Central University, Chung-Li, Taiwan

C. Adloff ${ }^{59}$, C.M. Kuo, W. Lin, A. Roy, T. Sarkar ${ }^{34}$, S.S. Yu

National Taiwan University (NTU), Taipei, Taiwan

L. Ceard, P. Chang, Y. Chao, K.F. Chen, P.H. Chen, W.-S. Hou, Y.y. Li, R.-S. Lu,

E. Paganis, A. Psallidas, A. Steen, E. Yazgan

Chulalongkorn University, Faculty of Science, Department of Physics, Bangkok, Thailand

B. Asavapibhop, C. Asawatangtrakuldee, N. Srimanobhas

Çukurova University, Physics Department, Science and Art Faculty, Adana, Turkey

F. Boran, S. Damarseckin ${ }^{60}$, Z.S. Demiroglu, F. Dolek, C. Dozen ${ }^{61}$, I. Dumanoglu ${ }^{62}$, E. Eskut, G. Gokbulut, Y. Guler, E. Gurpinar Guler ${ }^{63}$, I. $\operatorname{Hos}^{64}$, C. Isik, E.E. Kangal ${ }^{65}$, O. Kara, A. Kayis Topaksu, U. Kiminsu, G. Onengut, K. Ozdemir ${ }^{66}$, A. Polatoz, A.E. Simsek, B. Tali ${ }^{67}$, U.G. Tok, S. Turkcapar, I.S. Zorbakir, C. Zorbilmez

Middle East Technical University, Physics Department, Ankara, Turkey B. Isildak ${ }^{68}$, G. Karapinar ${ }^{69}$, K. Ocalan ${ }^{70}$, M. Yalvac ${ }^{71}$

Bogazici University, Istanbul, Turkey

I.O. Atakisi, E. Gülmez, M. Kaya ${ }^{72}$, O. Kaya ${ }^{73}$, Ö. Özçelik, S. Tekten ${ }^{74}$, E.A. Yetkin ${ }^{75}$

Istanbul Technical University, Istanbul, Turkey

A. Cakir, K. Cankocak ${ }^{62}$, Y. Komurcu, S. Sen ${ }^{76}$

Istanbul University, Istanbul, Turkey

F. Aydogmus Sen, S. Cerci ${ }^{67}$, B. Kaynak, S. Ozkorucuklu, D. Sunar Cerci ${ }^{67}$

Institute for Scintillation Materials of National Academy of Science of Ukraine, Kharkov, Ukraine

B. Grynyov

National Scientific Center, Kharkov Institute of Physics and Technology, Kharkov, Ukraine

L. Levchuk

University of Bristol, Bristol, United Kingdom

E. Bhal, S. Bologna, J.J. Brooke, E. Clement, D. Cussans, H. Flacher, J. Goldstein, G.P. Heath, H.F. Heath, L. Kreczko, B. Krikler, S. Paramesvaran, T. Sakuma, S. Seif El Nasr-Storey, V.J. Smith, J. Taylor, A. Titterton

Rutherford Appleton Laboratory, Didcot, United Kingdom

K.W. Bell, A. Belyaev ${ }^{77}$, C. Brew, R.M. Brown, D.J.A. Cockerill, K.V. Ellis, K. Harder, S. Harper, J. Linacre, K. Manolopoulos, D.M. Newbold, E. Olaiya, D. Petyt, T. Reis, T. Schuh, C.H. Shepherd-Themistocleous, A. Thea, I.R. Tomalin, T. Williams 


\section{Imperial College, London, United Kingdom}

R. Bainbridge, P. Bloch, S. Bonomally, J. Borg, S. Breeze, O. Buchmuller, A. Bundock, V. Cepaitis, G.S. Chahal ${ }^{78}$, D. Colling, P. Dauncey, G. Davies, M. Della Negra, P. Everaerts, G. Fedi, G. Hall, G. Iles, J. Langford, L. Lyons, A.-M. Magnan, S. Malik, A. Martelli, V. Milosevic, J. Nash ${ }^{79}$, V. Palladino, M. Pesaresi, D.M. Raymond, A. Richards, A. Rose, E. Scott, C. Seez, A. Shtipliyski, M. Stoye, A. Tapper, K. Uchida, T. Virdee ${ }^{19}$, N. Wardle, S.N. Webb, D. Winterbottom, A.G. Zecchinelli, S.C. Zenz

\section{Brunel University, Uxbridge, United Kingdom}

J.E. Cole, P.R. Hobson, A. Khan, P. Kyberd, C.K. Mackay, I.D. Reid, L. Teodorescu, S. Zahid

\section{Baylor University, Waco, U.S.A.}

A. Brinkerhoff, K. Call, B. Caraway, J. Dittmann, K. Hatakeyama, A.R. Kanuganti, C. Madrid, B. McMaster, N. Pastika, S. Sawant, C. Smith

Catholic University of America, Washington, DC, U.S.A.

R. Bartek, A. Dominguez, R. Uniyal, A.M. Vargas Hernandez

The University of Alabama, Tuscaloosa, U.S.A.

A. Buccilli, O. Charaf, S.I. Cooper, S.V. Gleyzer, C. Henderson, P. Rumerio, C. West

Boston University, Boston, U.S.A.

A. Akpinar, A. Albert, D. Arcaro, C. Cosby, Z. Demiragli, D. Gastler, C. Richardson, J. Rohlf, K. Salyer, D. Sperka, D. Spitzbart, I. Suarez, S. Yuan, D. Zou

\section{Brown University, Providence, U.S.A.}

G. Benelli, B. Burkle, X. Coubez ${ }^{20}$, D. Cutts, Y.t. Duh, M. Hadley, U. Heintz, J.M. $\operatorname{Hogan}^{80}$, K.H.M. Kwok, E. Laird, G. Landsberg, K.T. Lau, J. Lee, M. Narain, S. Sagir ${ }^{81}$, R. Syarif, E. Usai, W.Y. Wong, D. Yu, W. Zhang

University of California, Davis, Davis, U.S.A.

R. Band, C. Brainerd, R. Breedon, M. Calderon De La Barca Sanchez, M. Chertok, J. Conway, R. Conway, P.T. Cox, R. Erbacher, C. Flores, G. Funk, F. Jensen, W. Ko ${ }^{\dagger}$, O. Kukral, R. Lander, M. Mulhearn, D. Pellett, J. Pilot, M. Shi, D. Taylor, K. Tos, M. Tripathi, Y. Yao, F. Zhang

University of California, Los Angeles, U.S.A.

M. Bachtis, R. Cousins, A. Dasgupta, A. Florent, D. Hamilton, J. Hauser, M. Ignatenko, T. Lam, N. Mccoll, W.A. Nash, S. Regnard, D. Saltzberg, C. Schnaible, B. Stone, V. Valuev

University of California, Riverside, Riverside, U.S.A.

K. Burt, Y. Chen, R. Clare, J.W. Gary, S.M.A. Ghiasi Shirazi, G. Hanson, G. Karapostoli, O.R. Long, N. Manganelli, M. Olmedo Negrete, M.I. Paneva, W. Si, S. Wimpenny, Y. Zhang 
University of California, San Diego, La Jolla, U.S.A.

J.G. Branson, P. Chang, S. Cittolin, S. Cooperstein, N. Deelen, M. Derdzinski, J. Duarte, R. Gerosa, D. Gilbert, B. Hashemi, D. Klein, V. Krutelyov, J. Letts, M. Masciovecchio, S. May, S. Padhi, M. Pieri, V. Sharma, M. Tadel, F. Würthwein, A. Yagil

University of California, Santa Barbara - Department of Physics, Santa Barbara, U.S.A.

N. Amin, C. Campagnari, M. Citron, A. Dorsett, V. Dutta, J. Incandela, B. Marsh, H. Mei, A. Ovcharova, H. Qu, M. Quinnan, J. Richman, U. Sarica, D. Stuart, S. Wang

California Institute of Technology, Pasadena, U.S.A.

D. Anderson, A. Bornheim, O. Cerri, I. Dutta, J.M. Lawhorn, N. Lu, J. Mao, H.B. Newman, T.Q. Nguyen, J. Pata, M. Spiropulu, J.R. Vlimant, S. Xie, Z. Zhang, R.Y. Zhu

Carnegie Mellon University, Pittsburgh, U.S.A.

J. Alison, M.B. Andrews, T. Ferguson, T. Mudholkar, M. Paulini, M. Sun, I. Vorobiev

University of Colorado Boulder, Boulder, U.S.A.

J.P. Cumalat, W.T. Ford, E. MacDonald, T. Mulholland, R. Patel, A. Perloff, K. Stenson, K.A. Ulmer, S.R. Wagner

Cornell University, Ithaca, U.S.A.

J. Alexander, Y. Cheng, J. Chu, D.J. Cranshaw, A. Datta, A. Frankenthal, K. Mcdermott, J. Monroy, J.R. Patterson, D. Quach, A. Ryd, W. Sun, S.M. Tan, Z. Tao, J. Thom, P. Wittich, M. Zientek

Fermi National Accelerator Laboratory, Batavia, U.S.A.

S. Abdullin, M. Albrow, M. Alyari, G. Apollinari, A. Apresyan, A. Apyan, S. Banerjee, L.A.T. Bauerdick, A. Beretvas, D. Berry, J. Berryhill, P.C. Bhat, K. Burkett, J.N. Butler, A. Canepa, G.B. Cerati, H.W.K. Cheung, F. Chlebana, M. Cremonesi, V.D. Elvira, J. Freeman, Z. Gecse, E. Gottschalk, L. Gray, D. Green, S. Grünendahl, O. Gutsche, R.M. Harris, S. Hasegawa, R. Heller, T.C. Herwig, J. Hirschauer, B. Jayatilaka, S. Jindariani, M. Johnson, U. Joshi, T. Klijnsma, B. Klima, M.J. Kortelainen, S. Lammel, J. Lewis, D. Lincoln, R. Lipton, M. Liu, T. Liu, J. Lykken, K. Maeshima, D. Mason, P. McBride, P. Merkel, S. Mrenna, S. Nahn, V. O'Dell, V. Papadimitriou, K. Pedro, C. Pena ${ }^{50}$, O. Prokofyev, F. Ravera, A. Reinsvold Hall, L. Ristori, B. Schneider, E. SextonKennedy, N. Smith, A. Soha, W.J. Spalding, L. Spiegel, S. Stoynev, J. Strait, L. Taylor, S. Tkaczyk, N.V. Tran, L. Uplegger, E.W. Vaandering, M. Wang, H.A. Weber, A. Woodard

University of Florida, Gainesville, U.S.A.

D. Acosta, P. Avery, D. Bourilkov, L. Cadamuro, V. Cherepanov, F. Errico, R.D. Field, D. Guerrero, B.M. Joshi, M. Kim, J. Konigsberg, A. Korytov, K.H. Lo, K. Matchev, N. Menendez, G. Mitselmakher, D. Rosenzweig, K. Shi, J. Wang, S. Wang, X. Zuo

Florida International University, Miami, U.S.A.

Y.R. Joshi 
Florida State University, Tallahassee, U.S.A.

T. Adams, A. Askew, D. Diaz, R. Habibullah, S. Hagopian, V. Hagopian, K.F. Johnson, R. Khurana, T. Kolberg, G. Martinez, H. Prosper, C. Schiber, R. Yohay, J. Zhang

Florida Institute of Technology, Melbourne, U.S.A.

M.M. Baarmand, S. Butalla, T. Elkafrawy ${ }^{15}$, M. Hohlmann, D. Noonan, M. Rahmani, M. Saunders, F. Yumiceva

University of Illinois at Chicago (UIC), Chicago, U.S.A.

M.R. Adams, L. Apanasevich, H. Becerril Gonzalez, R. Cavanaugh, X. Chen, S. Dittmer, O. Evdokimov, C.E. Gerber, D.A. Hangal, D.J. Hofman, C. Mills, G. Oh, T. Roy, M.B. Tonjes, N. Varelas, J. Viinikainen, H. Wang, X. Wang, Z. Wu

The University of Iowa, Iowa City, U.S.A.

M. Alhusseini, B. Bilki ${ }^{63}$, K. Dilsiz ${ }^{82}$, S. Durgut, R.P. Gandrajula, M. Haytmyradov, V. Khristenko, O.K. Köseyan, J.-P. Merlo, A. Mestvirishvili ${ }^{83}$, A. Moeller, J. Nachtman, H. Ogul ${ }^{84}$, Y. Onel, F. Ozok ${ }^{85}$, A. Penzo, C. Snyder, E. Tiras, J. Wetzel, K. Yi ${ }^{86}$

Johns Hopkins University, Baltimore, U.S.A.

O. Amram, B. Blumenfeld, L. Corcodilos, M. Eminizer, A.V. Gritsan, S. Kyriacou, P. Maksimovic, C. Mantilla, J. Roskes, M. Swartz, T.Á. Vámi

The University of Kansas, Lawrence, U.S.A.

C. Baldenegro Barrera, P. Baringer, A. Bean, A. Bylinkin, T. Isidori, S. Khalil, J. King, G. Krintiras, A. Kropivnitskaya, C. Lindsey, N. Minafra, M. Murray, C. Rogan, C. Royon, S. Sanders, E. Schmitz, J.D. Tapia Takaki, Q. Wang, J. Williams, G. Wilson

\section{Kansas State University, Manhattan, U.S.A.}

S. Duric, A. Ivanov, K. Kaadze, D. Kim, Y. Maravin, D.R. Mendis, T. Mitchell, A. Modak, A. Mohammadi

Lawrence Livermore National Laboratory, Livermore, U.S.A.

F. Rebassoo, D. Wright

University of Maryland, College Park, U.S.A.

E. Adams, A. Baden, O. Baron, A. Belloni, S.C. Eno, Y. Feng, N.J. Hadley, S. Jabeen, G.Y. Jeng, R.G. Kellogg, T. Koeth, A.C. Mignerey, S. Nabili, M. Seidel, A. Skuja, S.C. Tonwar, L. Wang, K. Wong

Massachusetts Institute of Technology, Cambridge, U.S.A.

D. Abercrombie, B. Allen, R. Bi, S. Brandt, W. Busza, I.A. Cali, Y. Chen, M. D'Alfonso, G. Gomez Ceballos, M. Goncharov, P. Harris, D. Hsu, M. Hu, M. Klute, D. Kovalskyi, J. Krupa, Y.-J. Lee, P.D. Luckey, B. Maier, A.C. Marini, C. Mcginn, C. Mironov, S. Narayanan, X. Niu, C. Paus, D. Rankin, C. Roland, G. Roland, Z. Shi, G.S.F. Stephans, K. Sumorok, K. Tatar, D. Velicanu, J. Wang, T.W. Wang, Z. Wang, B. Wyslouch 
University of Minnesota, Minneapolis, U.S.A.

R.M. Chatterjee, A. Evans, S. Guts ${ }^{\dagger}$, P. Hansen, J. Hiltbrand, Sh. Jain, M. Krohn, Y. Kubota, Z. Lesko, J. Mans, M. Revering, R. Rusack, R. Saradhy, N. Schroeder, N. Strobbe, M.A. Wadud

University of Mississippi, Oxford, U.S.A.

J.G. Acosta, S. Oliveros

University of Nebraska-Lincoln, Lincoln, U.S.A.

K. Bloom, S. Chauhan, D.R. Claes, C. Fangmeier, L. Finco, F. Golf, J.R. González Fernández, I. Kravchenko, J.E. Siado, G.R. Snow ${ }^{\dagger}$, B. Stieger, W. Tabb

State University of New York at Buffalo, Buffalo, U.S.A.

G. Agarwal, C. Harrington, L. Hay, I. Iashvili, A. Kharchilava, C. McLean, D. Nguyen, A. Parker, J. Pekkanen, S. Rappoccio, B. Roozbahani

Northeastern University, Boston, U.S.A.

G. Alverson, E. Barberis, C. Freer, Y. Haddad, A. Hortiangtham, G. Madigan, B. Marzocchi, D.M. Morse, V. Nguyen, T. Orimoto, L. Skinnari, A. Tishelman-Charny, T. Wamorkar, B. Wang, A. Wisecarver, D. Wood

Northwestern University, Evanston, U.S.A.

S. Bhattacharya, J. Bueghly, Z. Chen, A. Gilbert, T. Gunter, K.A. Hahn, N. Odell, M.H. Schmitt, K. Sung, M. Velasco

University of Notre Dame, Notre Dame, U.S.A.

R. Bucci, N. Dev, R. Goldouzian, M. Hildreth, K. Hurtado Anampa, C. Jessop, D.J. Karmgard, K. Lannon, W. Li, N. Loukas, N. Marinelli, I. Mcalister, F. Meng, K. Mohrman, Y. Musienko ${ }^{44}$, R. Ruchti, P. Siddireddy, S. Taroni, M. Wayne, A. Wightman, M. Wolf, L. Zygala

The Ohio State University, Columbus, U.S.A.

J. Alimena, B. Bylsma, B. Cardwell, L.S. Durkin, B. Francis, C. Hill, A. Lefeld, B.L. Winer, B.R. Yates

Princeton University, Princeton, U.S.A.

G. Dezoort, P. Elmer, B. Greenberg, N. Haubrich, S. Higginbotham, A. Kalogeropoulos, G. Kopp, S. Kwan, D. Lange, M.T. Lucchini, J. Luo, D. Marlow, K. Mei, I. Ojalvo, J. Olsen, C. Palmer, P. Piroué, D. Stickland, C. Tully

University of Puerto Rico, Mayaguez, U.S.A.

S. Malik, S. Norberg

Purdue University, West Lafayette, U.S.A.

V.E. Barnes, R. Chawla, S. Das, L. Gutay, M. Jones, A.W. Jung, B. Mahakud, G. Negro, N. Neumeister, C.C. Peng, S. Piperov, H. Qiu, J.F. Schulte, N. Trevisani, F. Wang, R. Xiao, W. Xie 
Purdue University Northwest, Hammond, U.S.A.

T. Cheng, J. Dolen, N. Parashar, M. Stojanovic

Rice University, Houston, U.S.A.

A. Baty, S. Dildick, K.M. Ecklund, S. Freed, F.J.M. Geurts, M. Kilpatrick, A. Kumar, W. Li, B.P. Padley, R. Redjimi, J. Roberts ${ }^{\dagger}$, J. Rorie, W. Shi, A.G. Stahl Leiton, A. Zhang

University of Rochester, Rochester, U.S.A.

A. Bodek, P. de Barbaro, R. Demina, J.L. Dulemba, C. Fallon, T. Ferbel, M. Galanti, A. Garcia-Bellido, O. Hindrichs, A. Khukhunaishvili, E. Ranken, R. Taus

Rutgers, The State University of New Jersey, Piscataway, U.S.A.

B. Chiarito, J.P. Chou, A. Gandrakota, Y. Gershtein, E. Halkiadakis, A. Hart, M. Heindl, E. Hughes, S. Kaplan, O. Karacheban ${ }^{23}$, I. Laflotte, A. Lath, R. Montalvo, K. Nash, M. Osherson, S. Salur, S. Schnetzer, S. Somalwar, R. Stone, S.A. Thayil, S. Thomas

University of Tennessee, Knoxville, U.S.A.

H. Acharya, A.G. Delannoy, S. Spanier

Texas A\&M University, College Station, U.S.A.

O. Bouhali ${ }^{87}$, M. Dalchenko, A. Delgado, R. Eusebi, J. Gilmore, T. Huang, T. Kamon ${ }^{88}$, H. Kim, S. Luo, S. Malhotra, R. Mueller, D. Overton, L. Perniè, D. Rathjens, A. Safonov, J. Sturdy

Texas Tech University, Lubbock, U.S.A.

N. Akchurin, J. Damgov, V. Hegde, S. Kunori, K. Lamichhane, S.W. Lee, T. Mengke, S. Muthumuni, T. Peltola, S. Undleeb, I. Volobouev, Z. Wang, A. Whitbeck

Vanderbilt University, Nashville, U.S.A.

E. Appelt, S. Greene, A. Gurrola, R. Janjam, W. Johns, C. Maguire, A. Melo, H. Ni, K. Padeken, F. Romeo, P. Sheldon, S. Tuo, J. Velkovska, M. Verweij

University of Virginia, Charlottesville, U.S.A.

L. Ang, M.W. Arenton, B. Cox, G. Cummings, J. Hakala, R. Hirosky, M. Joyce, A. Ledovskoy, C. Neu, B. Tannenwald, Y. Wang, E. Wolfe, F. Xia

Wayne State University, Detroit, U.S.A.

P.E. Karchin, N. Poudyal, P. Thapa

University of Wisconsin - Madison, Madison, WI, U.S.A.

K. Black, T. Bose, J. Buchanan, C. Caillol, S. Dasu, I. De Bruyn, C. Galloni, H. He, M. Herndon, A. Hervé, U. Hussain, A. Lanaro, A. Loeliger, R. Loveless, J. Madhusudanan Sreekala, A. Mallampalli, D. Pinna, T. Ruggles, A. Savin, V. Shang, V. Sharma, W.H. Smith, D. Teague, S. Trembath-reichert, W. Vetens

†: Deceased

1: Also at Vienna University of Technology, Vienna, Austria

2: Also at Department of Basic and Applied Sciences, Faculty of Engineering, Arab Academy for Science, Technology and Maritime Transport, Alexandria, Egypt

3: Also at Université Libre de Bruxelles, Bruxelles, Belgium 
4: Also at IRFU, CEA, Université Paris-Saclay, Gif-sur-Yvette, France

5: Also at Universidade Estadual de Campinas, Campinas, Brazil

6: Also at Federal University of Rio Grande do Sul, Porto Alegre, Brazil

7: Also at UFMS, Nova Andradina, Brazil

8: Also at Universidade Federal de Pelotas, Pelotas, Brazil

9: Also at University of Chinese Academy of Sciences, Beijing, China

10: Also at Institute for Theoretical and Experimental Physics named by A.I. Alikhanov of NRC 'Kurchatov Institute', Moscow, Russia

11: Also at Joint Institute for Nuclear Research, Dubna, Russia

12: Also at Cairo University, Cairo, Egypt

13: Also at Zewail City of Science and Technology, Zewail, Egypt

14: Also at British University in Egypt, Cairo, Egypt

15: Now at Ain Shams University, Cairo, Egypt

16: Also at Purdue University, West Lafayette, U.S.A.

17: Also at Université de Haute Alsace, Mulhouse, France

18: Also at Erzincan Binali Yildirim University, Erzincan, Turkey

19: Also at CERN, European Organization for Nuclear Research, Geneva, Switzerland

20: Also at RWTH Aachen University, III. Physikalisches Institut A, Aachen, Germany

21: Also at University of Hamburg, Hamburg, Germany

22: Also at Department of Physics, Isfahan University of Technology, Isfahan, Iran, Isfahan, Iran

23: Also at Brandenburg University of Technology, Cottbus, Germany

24: Also at Skobeltsyn Institute of Nuclear Physics, Lomonosov Moscow State University, Moscow, Russia

25: Also at Institute of Physics, University of Debrecen, Debrecen, Hungary, Debrecen, Hungary

26: Also at Physics Department, Faculty of Science, Assiut University, Assiut, Egypt

27: Also at MTA-ELTE Lendület CMS Particle and Nuclear Physics Group, Eötvös Loránd University, Budapest, Hungary, Budapest, Hungary

28: Also at Institute of Nuclear Research ATOMKI, Debrecen, Hungary

29: Also at IIT Bhubaneswar, Bhubaneswar, India, Bhubaneswar, India

30: Also at Institute of Physics, Bhubaneswar, India

31: Also at G.H.G. Khalsa College, Punjab, India

32: Also at Shoolini University, Solan, India

33: Also at University of Hyderabad, Hyderabad, India

34: Also at University of Visva-Bharati, Santiniketan, India

35: Also at Indian Institute of Technology (IIT), Mumbai, India

36: Also at Deutsches Elektronen-Synchrotron, Hamburg, Germany

37: Also at Department of Physics, University of Science and Technology of Mazandaran, Behshahr, Iran

38: Now at INFN Sezione di $\operatorname{Bari}^{a}$, Università di Bari ${ }^{b}$, Politecnico di $\operatorname{Bari}^{c}$, Bari, Italy

39: Also at Italian National Agency for New Technologies, Energy and Sustainable Economic Development, Bologna, Italy

40: Also at Centro Siciliano di Fisica Nucleare e di Struttura Della Materia, Catania, Italy

41: Also at Riga Technical University, Riga, Latvia, Riga, Latvia

42: Also at Consejo Nacional de Ciencia y Tecnología, Mexico City, Mexico

43: Also at Warsaw University of Technology, Institute of Electronic Systems, Warsaw, Poland

44: Also at Institute for Nuclear Research, Moscow, Russia

45: Now at National Research Nuclear University 'Moscow Engineering Physics Institute' (MEPhI), Moscow, Russia 
46: Also at St. Petersburg State Polytechnical University, St. Petersburg, Russia

47: Also at University of Florida, Gainesville, U.S.A.

48: Also at Imperial College, London, United Kingdom

49: Also at P.N. Lebedev Physical Institute, Moscow, Russia

50: Also at California Institute of Technology, Pasadena, U.S.A.

51: Also at Budker Institute of Nuclear Physics, Novosibirsk, Russia

52: Also at Faculty of Physics, University of Belgrade, Belgrade, Serbia

53: Also at Università degli Studi di Siena, Siena, Italy

54: Also at Trincomalee Campus, Eastern University, Sri Lanka, Nilaveli, Sri Lanka

55: Also at INFN Sezione di Pavia ${ }^{a}$, Università di Pavia ${ }^{b}$, Pavia, Italy, Pavia, Italy

56: Also at National and Kapodistrian University of Athens, Athens, Greece

57: Also at Universität Zürich, Zurich, Switzerland

58: Also at Stefan Meyer Institute for Subatomic Physics, Vienna, Austria, Vienna, Austria

59: Also at Laboratoire d'Annecy-le-Vieux de Physique des Particules, IN2P3-CNRS, Annecyle-Vieux, France

60: Also at Şrnak University, Sirnak, Turkey

61: Also at Department of Physics, Tsinghua University, Beijing, China, Beijing, China

62: Also at Near East University, Research Center of Experimental Health Science, Nicosia, Turkey

63: Also at Beykent University, Istanbul, Turkey, Istanbul, Turkey

64: Also at Istanbul Aydin University, Application and Research Center for Advanced Studies (App. \& Res. Cent. for Advanced Studies), Istanbul, Turkey

65: Also at Mersin University, Mersin, Turkey

66: Also at Piri Reis University, Istanbul, Turkey

67: Also at Adiyaman University, Adiyaman, Turkey

68: Also at Ozyegin University, Istanbul, Turkey

69: Also at Izmir Institute of Technology, Izmir, Turkey

70: Also at Necmettin Erbakan University, Konya, Turkey

71: Also at Bozok Universitetesi Rektörlügü, Yozgat, Turkey

72: Also at Marmara University, Istanbul, Turkey

73: Also at Milli Savunma University, Istanbul, Turkey

74: Also at Kafkas University, Kars, Turkey

75: Also at Istanbul Bilgi University, Istanbul, Turkey

76: Also at Hacettepe University, Ankara, Turkey

77: Also at School of Physics and Astronomy, University of Southampton, Southampton, United Kingdom

78: Also at IPPP Durham University, Durham, United Kingdom

79: Also at Monash University, Faculty of Science, Clayton, Australia

80: Also at Bethel University, St. Paul, Minneapolis, U.S.A., St. Paul, U.S.A.

81: Also at Karamanoğlu Mehmetbey University, Karaman, Turkey

82: Also at Bingol University, Bingol, Turkey

83: Also at Georgian Technical University, Tbilisi, Georgia

84: Also at Sinop University, Sinop, Turkey

85: Also at Mimar Sinan University, Istanbul, Istanbul, Turkey

86: Also at Nanjing Normal University Department of Physics, Nanjing, China

87: Also at Texas A\&M University at Qatar, Doha, Qatar

88: Also at Kyungpook National University, Daegu, Korea, Daegu, Korea 